(C) 2018 Universidad Nacional Autónoma de México, Facultad de Estudios Superiores Zaragoza.

Este es un artículo Open Access bajo la licencia CC BY-NC-ND (http://creativecommons.org/licenses/by-nc-nd/4.0/).

TIP Revista Especializada en Ciencias Químico-Biológicas, 21(Supl. 2): 13-29, 2018.

DOI: 10.22201/fesz.23958723e.2018.0.157

\title{
RELACIÓN ENTRE SUCESIÓN ECOLÓGICA VEGETAL Y HONGOS MICORRIZÓGENOS ARBUSCULARES EN un MATORRAL XERÓFILO EN EL CENTRO DE MÉXICO
}

\author{
Arcadio Monroy-Ata' y Karen Yadira Ramírez-Saldívar \\ Unidad de Investigación en Ecología Vegetal, Facultad de Estudios Superiores Zaragoza, \\ Universidad Nacional Autónoma de México, Av. Guelatao \# 66, Col. Ejército de Oriente, \\ Ciudad de México, 09230, México. E-mails: arcadiom@unam.mx,rmzbio@gmail.com
}

\begin{abstract}
Resumen
El objetivo fue determinar la relación entre la estructura del mosaico vegetal de cuatro etapas serales de un matorral xerófilo, caracterizadas respectivamente por Buchloë dactyloides, Mimosa depauperata, M. biuncifera y Prosopis laevigata, durante la maduración del ecosistema, respecto a la formación de nuevos estratos con plantas dominantes de mayor altura que en la etapa anterior, y la estructura de la comunidad de hongos micorrizógenos arbusculares (HMA) de cada fase sucesional. Para esto, se determinaron las morfo-especies y el porcentaje de colonización micorrízica de los HMA asociados a gramíneas establecidas en las cuatro etapas serales. También, se caracterizó la comunidad vegetal de las cuatro fases sucesionales en un agostadero semiárido del Valle del Mezquital, Hidalgo, en el Centro de México. Se calculó el Índice de Valor de Importancia para las especies de cada mosaico vegetal y el Índice de diversidad de Shannon-Wiener para la vegetación y para las morfo-especies de HMA, en cada etapa seral. En la colonización micorrízica de las raíces, el mosaico vegetal dominado por $M$. depauperata tuvo el porcentaje más alto (88\%) y el dominado por B. dactyloides tuvo la menor colonización (67\%). También, se realizó una comparación de las curvas de distribución de abundancias relativas entre especies de las comunidades vegetales y las morfo-especies de HMA y en las cuatro etapas serales se encontró un ajuste de curvas estadísticamente significativo, mediante la prueba de chi cuadrada. Esto muestra una correspondencia entre estructuras comunitarias de fitobiontes y micobiontes, donde las plantas nodriza tienen un papel determinante en la organización de los gremios vegetales. Se concluye que hay una estrecha relación entre la comunidad de HMA y las especies de los mosaicos vegetales, ya que ambas tienen el mismo patrón de dominancias específicas en cada etapa seral, en el matorral xerófilo analizado. No se encontraron diferencias significativas en los porcentajes de colonización micorrízica radical durante la maduración del ecosistema, lo que indica que hay una comunidad de HMA desde la fase sucesional temprana; esto muestra un papel central de las plantas nodriza y de su red hifal en el proceso de sucesión ecológica en ambientes semiáridos.

Palabras Clave: sucesión ecológica, matorral xerófilo, hongos micorrizógenos arbusculares, colonización micorrízica, distribución de abundancias relativas de especies.
\end{abstract}

\section{Relation between plant ecological succession and arbuscular mycorrhizal fungi in a xeric shrub of Central Mexico}

\begin{abstract}
The objective was to determine the relation between plant guild structure of four successional phases in a xeric shrub, dominated respectively by Buchloë dactyloides, Mimosa depauperata, M. biuncifera and Prosopis laevigata, during ecosystem maturation, with respect to new stratifications with dominant plants of bigger height that in the previous phase, and the community structure of arbuscular mycorrhizal fungi (AMF) in each successional phase. For this, it was determinated the morpho-species and percentage of mycorrhizal colonization of AMF associated with established grass individuals, in the four plant guilds. Also, it was characterized the plant community of the four successional phases in a semiarid rangeland at the Mezquital Valley, Hidalgo State, in Central Mexico. In the same way, it was calculated the Importance Value Index for the species of each plant guild and the Shannon-Wiener diversity index for the plant community and for the AMF morpho-species for each successional phase. For the mycorrhizal root colonization, the plant guild dominated by_M. depauperata had the highest percentage (88\%) and B. dactyloides had the lowest colonization (67\%). Also, it was made a comparison of the relative abundance distribution (RAD) curves between the species of plant guilds and the AMF morpho-species and in the four successional phases it was found a fitting of the RAD curves, statistically significant with square chi tests. This shows a correspondence between community structures of phytobionts and mycobionts, where the nurse plants have a determinant role in plant guild organization. It was concluded that there is a narrow relation between the AMF community and the plant guild species, since both communities have the same specific dominance pattern for each successional phase, in the xeric shrub analyzed. There were no significant differences in the root mycorrhizal colonization percentages during ecosystem maturation, indicating that there is an AMF community from the early phase; this shows a central role of the nurse plants and their hyphal network in the ecological succession process in semiarid environments.

Key words: ecological succession, xeric shrub, arbuscular mycorrhizal fungi, mycorrhizal colonization, specific relative abundance distribution.
\end{abstract}

Nota: Artículo recibido el 30 de agosto del 2017 y aceptado el 18 de octubre del 2018. 


\section{INTRODUCCIÓN}

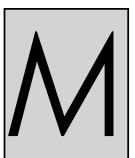

éxico es un país cuyo territorio presenta extensas regiones de zonas áridas y semiáridas, que cubren el $54.3 \%$ de su superficie total (Cervantes, 2002). El semidesierto mexicano es importante debido al alto número de especies vegetales endémicas (Siqueira, Carbone, Curi, Rosado \& Davide, 1998) y a que es el centro de diversificación de familias botánicas como Cactaceae, Crassulaceae y Agavaceae (Siqueira, Carbone, Curi, Rosado \& Davide, 1998; Miranda, 1955). La escasez de agua disponible para el desarrollo de las plantas es el principal factor limitante de la vegetación en estos ambientes, donde dominan los matorrales xerófilos (González, 2004) y la maduración de los ecosistemas transcurre con una marcada estacionalidad anual dividida en dos periodos: lluvias y secas.

La sucesión ecológica es un proceso de sustitución de una comunidad biótica por otra con una mayor complejidad estructural y funcional durante la maduración de un ecosistema, después de que éste es perturbado (sucesión secundaria) o cuando la comunidad biótica se desarrolla desde un nuevo sustrato (sucesión primaria) (Laska, 2001; Morrin, 1999; Pickett \& White, 1985).

Las transformaciones ecológicas que sufre un ecosistema en sucesión secundaria -y la velocidad con la que ocurren estos cambios- dependen de las características del disturbio (e.g. extensión, intensidad y frecuencia), de la disponibilidad de propágulos regenerativos (como semillas y plántulas, de origen local y foráneo), del ambiente biótico (i.e. depredadores, carnívoros, herbívoros, patógenos y parásitos, entre otros) y de las condiciones abióticas prevalecientes en el sitio perturbado (Ramírez-Lozano et al., 2013; van der Heijden, Boller, Wiemken \& Sanders, 1998; Pickett, Collins \& Armesto, 1987; Grubb, 1985).

Por otra parte, la asociación simbiótica entre plantas terrestres y hongos micorrízicos, denominada micorrizógenos, es cosmopolita y seguramente es el mutualismo más extendido en la naturaleza. Por ejemplo, Smith \& Read (1997), quienes clasifican a las micorrizas en 5 tipos, estiman que entre el 80 y $90 \%$ de las plantas terrestres tienen asociación con la llamada endomicorriza, formada por hongos micorrizógenos arbusculares (HMA) phylum Glomeromycota, los cuales no desarrollan la red de Hartig y colonizan intracelularmente la corteza de la raíz por medio de estructuras especializadas denominadas arbúsculos, que actúan como órganos de intercambio de nutrimentos entre la célula vegetal y el hongo (Aguilera, Olalde, Arriaga $\&$ Contreras, 2007). Asimismo, en la relación planta-hongo las primeras obtienen frecuentemente un mayor porcentaje de supervivencia, mayor tolerancia a la sequía, salinidad y altas temperaturas, un desarrollo vigoroso y un incremento en la capacidad de fijar nitrógeno (Luna, 2005; Peña, 2002; Varela \& Trejo, 2001; Escalona, Trejo, Rivera, Lara \& Rivera, 1998;
Garrido, Díaz, Escalona \& Trejo, 1998). A su vez, el hongo tiene un hábitat intrarradical y recibe carbohidratos derivados de la fotosíntesis, además de que las hifas son importantes en la conservación de los suelos al participar en la formación de agregados estables (Miller \& Jastrow, 1992; Allen, 1991; Harley \& Smith, 1983). Asimismo, el micobionte contribuye a mantener el balance hídrico de la planta, lo cual favorece el incremento de la tasa de fotosíntesis, promoviendo un aumento de la biomasa de la planta y del hongo (González, Monroy, García \& Orozco, 2005; Gupta \& Mukerji, 2000).

Por lo anterior, la determinación de los HMA presentes en distintos estadios de maduración de un ecosistema terrestre puede ser una herramienta con la que se describa el proceso sucesional de la vegetación (Siqueira, Carbone, Curi, Rosado \& Davide, 1998; Aziz, Sylvia \& Doren, 1995; Evans \& Miller, 1990). Johnson (2010) explica que el modelo de coadaptación establece que la asociación entre las plantas y los HMA ejercen una fuerza selectiva entre sí, de manera que el intercambio de recursos simbióticos se favorece cuando hay factores limitantes del suelo como agua y nutrimentos. También, los HMAjuegan un papel importante en el mantenimiento del equilibrio de los ecosistemas y en los procesos de sucesión ecológica (Allen, 1991; Allen \& Allen, 1984).

Asimismo, los principales problemas que se presentan para la vegetación en zonas áridas y semiáridas son la escasa disponibilidad de agua para el desarrollo vegetal. Por ello, los HMA son simbiontes mutualistas en la mayoría de las plantas vasculares terrestres, ya que éstos aumentan la capacidad de absorber agua y nutrimentos para la vegetación, lo cual incrementa sus posibilidades de establecimiento y desarrollo. Por ello, se llevó a cabo un estudio analizando sincrónicamente cuatro fases de desarrollo, en cuanto a estructuración de la comunidad vegetal, de un matorral xerófilo, representadas por cuatro tipos de mosaico vegetal, caracterizados a su vez y secuencialmente durante la maduración del matorral, por la presencia recurrente de herbáceas perennes, arbustos bajos, arbustos medianos y árboles y su asociación con comunidades de HMA. Las preguntas planteadas al inicio de este trabajo fueron: ¿Existe una correlación entre las comunidades de HMA y el ensamblaje de especies del mosaico vegetal, de cada una de las cuatro fases sucesionales de un matorral xerófilo? y ¿Se incrementa el porcentaje de colonización micorrízica total, de gramíneas asociadas a las plantas dominantes de cada una de esas cuatro fases sucesionales de un matorral xerófilo, al aumentar el estado de maduración de la comunidad vegetal?

\section{Materiales y Métodos}

Zona de estudio. El área de trabajo de campo se localiza en la zona semiárida que forma parte del municipio de Santiago de Anaya, en el Estado de Hidalgo (Figura 1), dentro del denominado Valle del Mezquital. Este municipio se encuentra localizado en el paralelo $20^{\circ} 23$ ' $31^{\prime \prime}$ longitud norte y el meridiano 


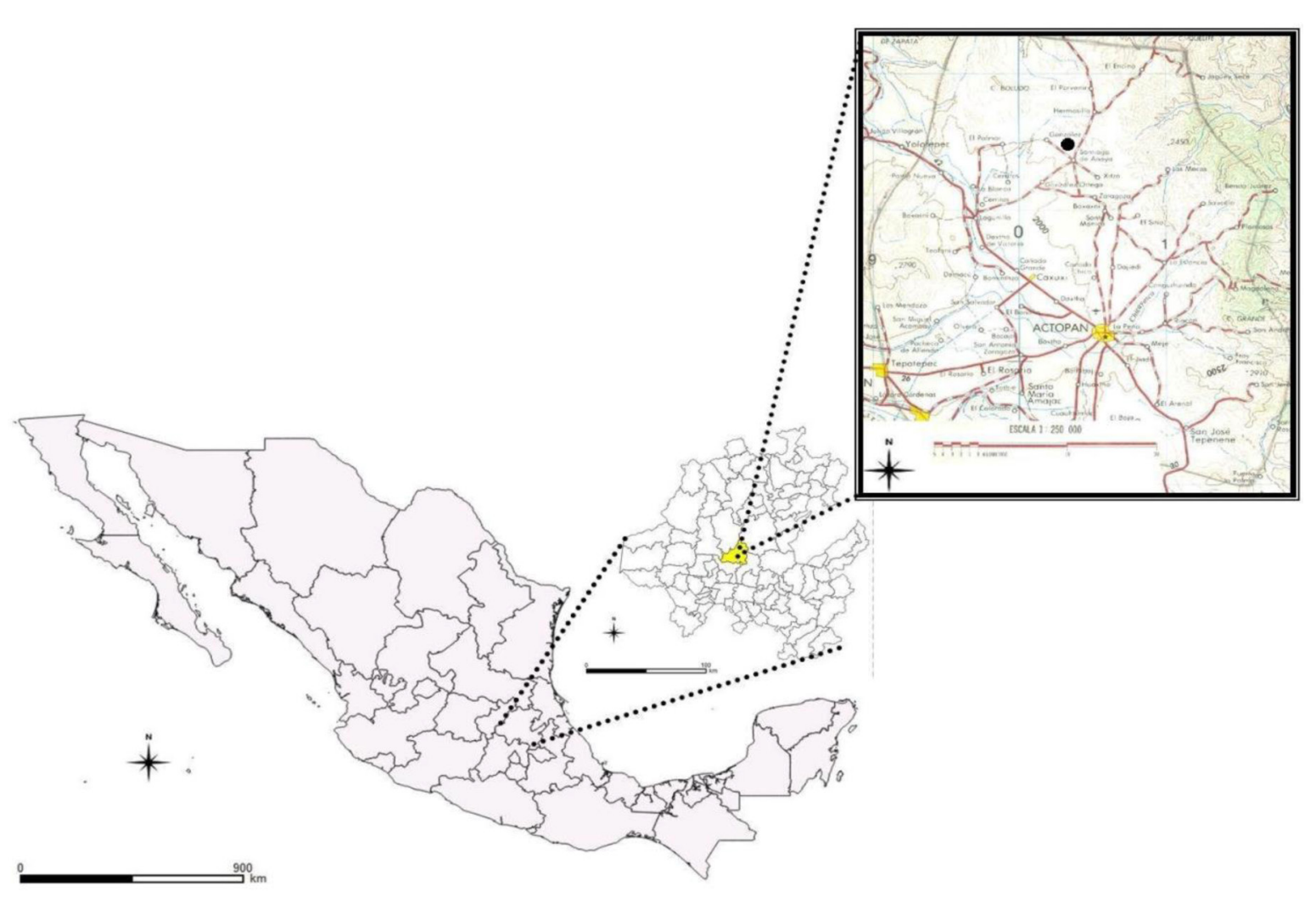

Figura 1. Mapa de la zona de muestreo; el punto en el recuadro de la derecha señala la zona de estudio, en el municipio de Santiago de Anaya, Hidalgo, localizado en el paralelo $20^{\circ} 23^{\prime} 31^{\prime \prime}$ longitud Norte y el meridiano $98^{\circ} 58^{\prime} 22^{\prime \prime}$ longitud Oeste (Fuente: GoogleEarth.com.mx).

$98^{\circ} 58^{\prime} 22^{\prime}$ 'longitud oeste, a una altitud de $2054 \mathrm{msnm}$. El clima es seco con lluvias en verano, con una temperatura y precipitación promedio anual de $24^{\circ} \mathrm{Cy} 550 \mathrm{~mm}$, respectivamente; la estación de lluvias ocurre de mayo a octubre y de noviembre a abril es la temporada seca (INEGI, 2000). El tipo de vegetación es matorral subinerme con dominancia de Flourensia resinosa y Mimosa depauperata. Es una zona semiárida donde las principales causas de deterioro son el sobrepastoreo, la tala de árboles y donde la mayoría de las especies palatables para el ganado ovino-caprino han sido desplazadas.

Fase de campo. Se tomaron datos de la vegetación a través del método de transectos con área (Mostacedo \& Fredericksen, 2000). Los mosaicos analizados fueron cuatro y corresponden a las plantas más conspicuas: 1) herbáceas perennes, 2) arbustos bajos $(<1 \mathrm{~m}), 3)$ arbustos medianos $(>1 \mathrm{~m})$ y 4) árboles (un solo fuste). Se realizaron dos repeticiones en cada uno de los cuatro mosaicos vegetales, los cuales fueron elegidos al azar. El muestreo se llevó a cabo formando un rectángulo de $20 \mathrm{~m}$ x $1 \mathrm{~m}$ donde se determinaron los siguientes atributos de vegetación: frecuencia, densidad, altura y cobertura. Las muestras de suelo con esporas de HMA fueron colectadas en la época seca, en los distintos mosaicos vegetales, dentro de los transectos seleccionados. Posteriormente se almacenó el material recolectado en bolsas de plástico y fue etiquetado; se colectaron cinco muestras por cada mosaico vegetal, obteniendo 20 muestras en total que se analizaron de forma independiente; estas muestras se colectaron a una profundidad de $20 \mathrm{~cm}$, bajo la cobertura de las plantas dominantes y cerca de las raíces de gramíneas, ya que éstas son masificadoras de esporas de HMA.

Perfil vertical. La estructura vertical se refiere a la disposición de las plantas de acuerdo con la composición de sus formas de vida en los diferentes estratos de la comunidad vegetal. Para la visualización de un perfil vertical fue necesaria la realización de diagramas gráficos de la vegetación. Esto se llevó a cabo a través de un levantamiento de la vegetación, tomando como parámetro principal la altura y la forma de la silueta de la vegetación (Mostacedo \& Fredericksen, 2000).

Riqueza de especies. La riqueza específica (S) se basa en el número de especies presentes, sin tomar en cuenta el valor de importancia de las mismas. Para esto se realizó una curva de acumulación de especies a partir del muestreo realizado en 
campo y una curva de rarefacción para estimar la diversidad alfa. La rarefacción implica construir una curva suavizada de acumulación de especies mediante un re-muestreo al azar de los datos. La curva fue realizada con el programa EstimateS 9 Windows (http:// viceroy.eeb.uconn.edu/estimates).

Índice de Valor de Importancia (IVI). Este parámetro se utilizó para el registro de la presencia y frecuencia de las especies y se calculó para cuatro mosaicos de vegetación, que representan cuatro fases sucesionales (colonización, intermedias tempranas, intermedias tardías y maduras) con dos repeticiones de cada uno, por lo que se obtuvieron datos de ocho unidades de muestreo (UM) (Cuadro 1). El IVI define cuáles de las especies presentes contribuyen en mayor medida al tipo de vegetación y a la estructura de un ecosistema (Cottam \& Curtis, 1956) y para este estudio se calculó utilizando la fórmula definida por Mueller \& Ellenberg (1974):

\section{IVI $=$ Dominancia relativa + Densidad relativa + Frecuencia relativa}

La dominancia relativa se calculó de la siguiente manera:

Dominancia relativa $=\frac{\text { Dominancia por cada especie }}{\text { Dominancia total para todas las especies }} \times 100$

donde:

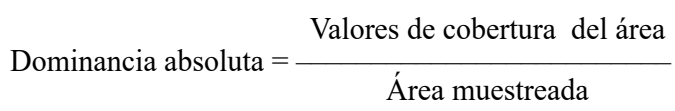

La densidad relativa se calculó de la siguiente manera:

$$
\text { Densidad relativa }=\frac{\text { Densidad absoluta por cada especie }}{\text { Densidad absoluta de todas las especies }} \times 100
$$

donde:

$$
\text { Densidad absoluta }=\frac{\text { Número de individuos de una especie }}{\text { Área muestreada }}
$$

La frecuencia relativa se calculó de la siguiente manera:

$$
\text { Frecuencia relativa }=\frac{\text { Frecuencia absoluta por cada especie }}{\text { Frecuencia absoluta de todas las especies }} \times 100
$$

\begin{tabular}{|c|c|c|}
\hline $\begin{array}{l}\text { Unidades de } \\
\text { Muestreo } \\
\text { (UM) }\end{array}$ & $\begin{array}{c}\text { Especie } \\
\text { característica }\end{array}$ & $\begin{array}{c}\text { Coordenadas del sitio de } \\
\text { muestreo }\end{array}$ \\
\hline 1 & \multirow[t]{2}{*}{$\begin{array}{c}\text { Buchlö̈ } \\
\text { dactyloides }\end{array}$} & $\begin{array}{c}\text { Latitud } 20^{\circ} 39^{\prime} 27^{\prime \prime} \\
\text { Longitud }-98^{\circ} 97^{\prime} 35^{\prime \prime}\end{array}$ \\
\hline 5 & & $\begin{array}{l}\text { Latitud } 20^{\circ} 39^{\prime} 26^{\prime \prime} \\
\text { Longitud }-98^{\circ} 97^{\prime} 34^{\prime \prime}\end{array}$ \\
\hline 2 & \multirow[t]{2}{*}{$\begin{array}{c}\text { Mimosa } \\
\text { depauperata }\end{array}$} & $\begin{array}{c}\text { Latitud } 20^{\circ} 39^{\prime} 28^{\prime \prime} \\
\text { Longitud }-98^{\circ} 97^{\prime} 37^{\prime \prime}\end{array}$ \\
\hline 6 & & $\begin{array}{c}\text { Latitud } 20^{\circ} 39^{\prime} 29^{\prime \prime} \\
\text { Longitud }-98^{\circ} 97^{\prime} 36^{\prime \prime}\end{array}$ \\
\hline 3 & \multirow[t]{2}{*}{$\begin{array}{l}\text { Mimosa } \\
\text { biuncifera }\end{array}$} & $\begin{array}{c}\text { Latitud } 20^{\circ} 39^{\prime} 32^{\prime \prime} \\
\text { Longitud }-98^{\circ} 97^{\prime} 41^{\prime \prime}\end{array}$ \\
\hline 7 & & $\begin{array}{c}\text { Latitud } 20^{\circ} 39^{\prime} 35^{\prime \prime} \\
\text { Longitud }-98^{\circ} 97^{\prime} 41^{\prime \prime}\end{array}$ \\
\hline 4 & \multirow[t]{2}{*}{$\begin{array}{l}\text { Prosopis } \\
\text { laevigata }\end{array}$} & $\begin{array}{c}\text { Latitud } 20^{\circ} 39^{\prime} 21^{\prime \prime} \\
\text { Longitud }-98^{\circ} 97^{\prime} 26^{\prime \prime}\end{array}$ \\
\hline 8 & & $\begin{array}{c}\text { Latitud } 20^{\circ} 39^{\prime} 45^{\prime \prime} \\
\text { Longitud }-98^{\circ} 97^{\prime} 23^{\prime \prime}\end{array}$ \\
\hline
\end{tabular}

donde:

$$
\text { Frecuencia absoluta }=\frac{\begin{array}{c}
\text { Número de cuadros en los que } \\
\text { se presenta cada especie }
\end{array}}{\text { Número total de cuadros muestreados }}
$$

Cuadro 1. Unidades de muestreo en el Valle del Mezquital, Hidalgo.

Índice de diversidad. Para el cálculo de la diversidad se utilizó el índice de Shannon-Wiener, la toma de datos se realizó mediante transectos elegidos de forma aleatoria. Este índice se calculó mediante la siguiente fórmula:

$$
H^{\prime}=-\Sigma p_{i} \ln p_{i}
$$

donde:

$H^{\prime}=$ Índice de Shannon-Wiener.

$p_{i}=$ Abundancia relativa.

$\ln =$ Logaritmo natural.

Extracción y determinación de esporas de HMA. La extracción de esporas se realizó mediante el método propuesto por Gerdemann \& Nicolson (1963). Posteriormente se observaron las esporas con ayuda de un estereoscopio, agrupándolas de acuerdo con sus características morfológicas como color, forma, tamaño y presencia/ausencia de hifas y finalmente se elaboraron preparaciones permanentes en alcohol polivinílicoácido láctico-glicerol (PVLG) más reactivo de Melzer para su determinación taxonómica, la cual se llevó a cabo con las descripciones de los diferentes géneros y especies de HMAque se encuentran disponibles en el International Culture Collection of (Vesicular) Arbuscular Mycorrhizal Fungi (INVAM http:// invam.caf.wvu.edu/). Las preparaciones fijas de las esporas de los HMA se encuentran disponibles para consulta en la Unidad de Investigación en Ecología Vegetal de la Facultad 
de Estudios Superiores Zaragoza, de la Universidad Nacional Autónoma de México.

Porcentaje de colonización micorrízica. El porcentaje de colonización se obtuvo a partir de raíces de gramíneas colectadas en los cuatro diferentes mosaicos vegetales, de la siguiente manera: para el mosaico de la etapa seral inicial se emplearon plantas de Buchloë dactyloides, y macollos de pasto ubicados bajo la cobertura de las plantas dominantes, en las otras tres fases sucesionales. En la fase de campo estas raíces se colocaron en frascos de vidrio con alcohol al 70\% para su transporte al laboratorio, guardándolas en el refrigerador para su posterior tinción con el método propuesto por Phillips \& Hayman (1970). La observación de las raíces, montadas en un portaobjetos, se realizó con ayuda de un microscopio compuesto; para esto, se observaron en la parte superior, media e inferior de cada preparación, con un total de 45 observaciones. El número de puntos colonizados anotados sobre el total de puntos observados de la relación micorrízica, se contabilizó como porcentaje utilizando la siguiente fórmula:

$$
\% \mathrm{C}=\mathrm{D} / \mathrm{T}(100)
$$

donde:

$\% \mathrm{C}=$ Porcentaje de colonización.

$\mathrm{D}=$ Presencia de vesículas, arbúsculos y/o hifas.

$\mathrm{T}=$ Campos totales observados.

Análisis de datos. Para determinar si había diferencias para el Índice de Valor de Importancia (IVI) y para el índice de Shannon-Wiener de la diversidad vegetal y las comunidades de HMA, entre los mosaicos de vegetación, los datos de cada variable se analizaron primero con una prueba de Normalidad (Shapiro-Wilks modificado; $\mathrm{p}<0.05$ ) basada en una muestra. Posteriormente, debido a que no se cumplió con el supuesto de normalidad y para determinar si existían diferencias significativas entre mosaicos vegetales, se aplicó la prueba no paramétrica de Kruskal Wallis $(\mathrm{p}<0.05)$. Los análisis fueron realizados con el programa InfoStat (2008) (Di Rienzo et al., 2016). Asimismo, para conocer si la diversidad de esporas de HMA y la diversidad de la vegetación en cada etapa seral mostraban correlación se realizaron gráficas de Distribución de la Abundancia Relativa tanto para la vegetación como para las comunidades de HMA (transformando estos datos mediante la obtención de su raíz cuadrada) y después se aplicó una prueba de $\chi_{i}^{2}$ (bondad de ajuste entre curvas).

\section{REsultados}

Perfil vertical. En la primer etapa seral (herbáceas perennes) caracterizada por Buchloë dactyloides, las especies vegetales presentes en esta comunidad, en orden de importancia, son: Mimosa depauperata, Buchloëdactyloides, Flourensia resinosa, gramínea 3, Mimosa biuncifera, Condalia mexicana, Agave salmiana, Opuntia cantabrigiensis (Figura 2). En la segunda etapa seral (arbustos bajos $<1 \mathrm{~m}$ ) dominada por Mimosa depauperata, las especies vegetales que caracterizan esta comunidad son en orden de importancia: Mimosa depauperata, Flourensia resinosa, Buchloë dactyloides, Agave salmiana, Opuntia cantabrigiensis, gramínea 3, Condalia mexicana, Opuntia imbricata, Coryphanta radians, gramíneas 1 y 2, Jatropha dioica y musgo (Figura 3). En la tercer etapa seral (arbustos medianos $>1 \mathrm{~m}$ ) con dominancia de Mimosa biuncifera, las especies vegetales que caracterizan esta comunidad son en orden de importancia: Mimosa biuncifera, Buchloë dactyloides, Flourensia resinosa, Condalia mexicana, Mimosa depauperata, gramínea 3, Prosopis laevigata, Opuntia cantabrigiensis, Opuntia imbricata, Agave salmiana, Coryphanta radians y Acaciafarnesiana (Figura 4) y en la cuarta etapa seral(árboles), dominada por Prosopis laevigata, las especies vegetales que caracterizan esta comunidad son en orden de importancia: Prosopis laevigata, Flourensia resinosa, Mimosa depauperata, Karwinskia humboldtiana, Agave salmiana, Condaliamexicana, Stenocereus sp., Opuntia streptacantha, Opuntia imbricata, y Tillandsia recurvata (Figura 5).

Curva de acumulación de especies. La Figura 6 es una curva de acumulación de especies (datos observados), la cual representa

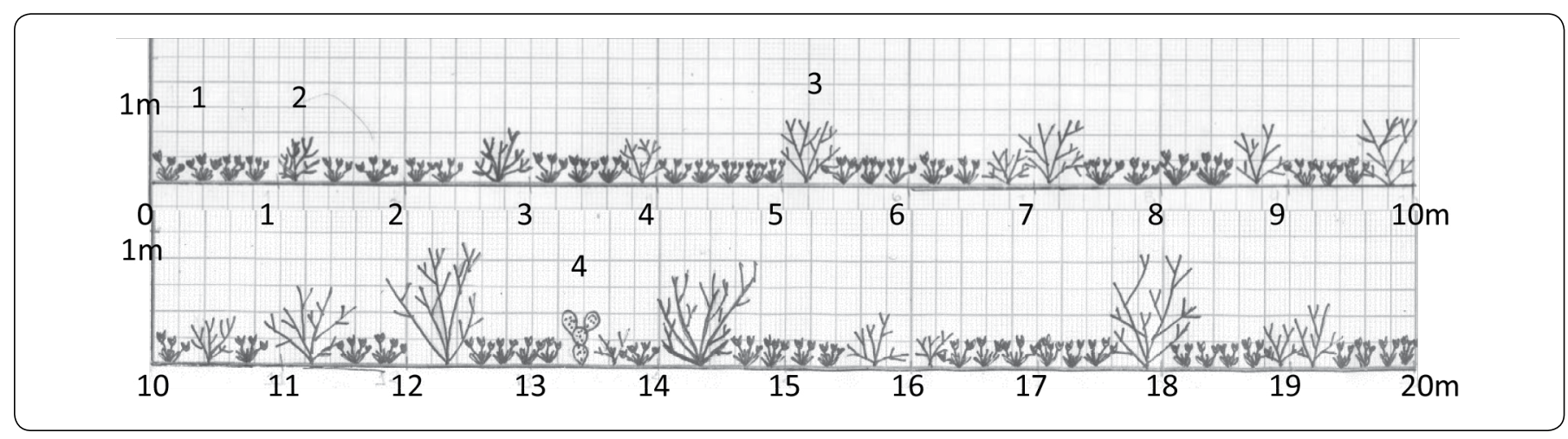

Figura 2. Perfil estructural vertical de la vegetación dominada por herbáceas perennes en un matorral xerófilo en el Valle del Mezquital, Hidalgo. 1 Buchloë dactyloides, 2 Flourensia resinosa, 3 Mimosa depauperata, 4 Opuntia cantabrigiensis. 

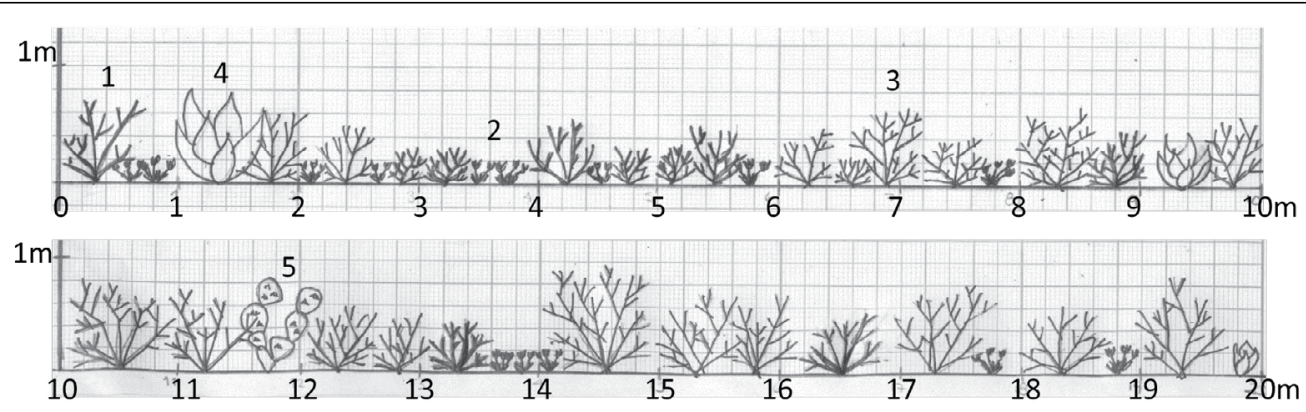

Figura 3. Perfil estructural vertical de la vegetación dominada por arbustos menores a $1 \mathrm{~m}$ en un matorral xerófilo en el Valle del Mezquital, Hidalgo. 1 Flourensia resinosa, 2 Buchloë dactyloides, 3 Mimosa depauperata, 4 Agave salmiana, 5 Opuntia cantabrigiensis.

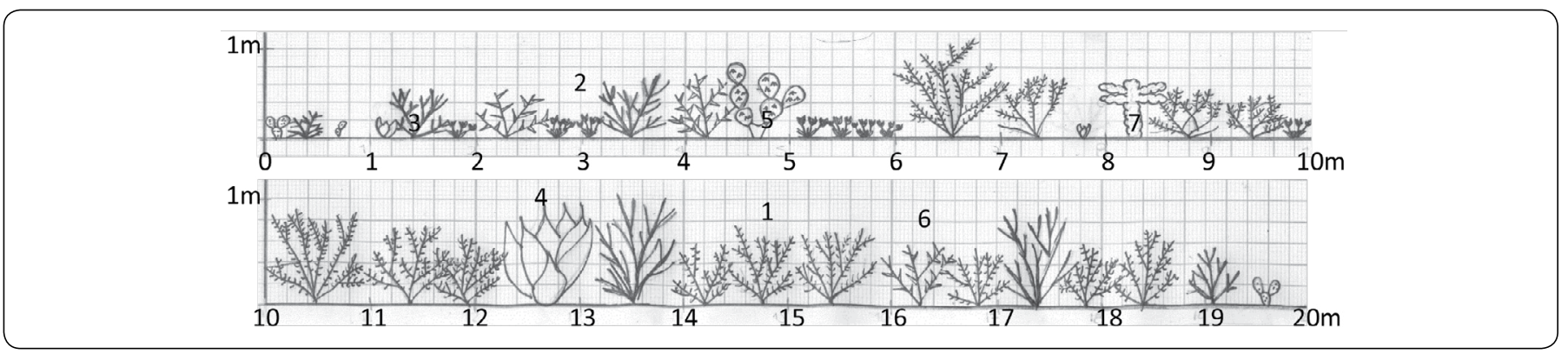

Figura 4. Perfil vertical de la vegetación dominada por arbustos medianos de talla mayor a $1 \mathrm{~m}$, en un matorral xerófilo en el Valle del Mezquital, Hidalgo. 1 Mimosa biuncifera, 2 Buchloë dactyloides, 3 Flourensia resinosa, 4 Agave salmiana, 5 Opuntia cantabrigiensis, 6 Condalia mexicana, 7 Opuntia imbricata.
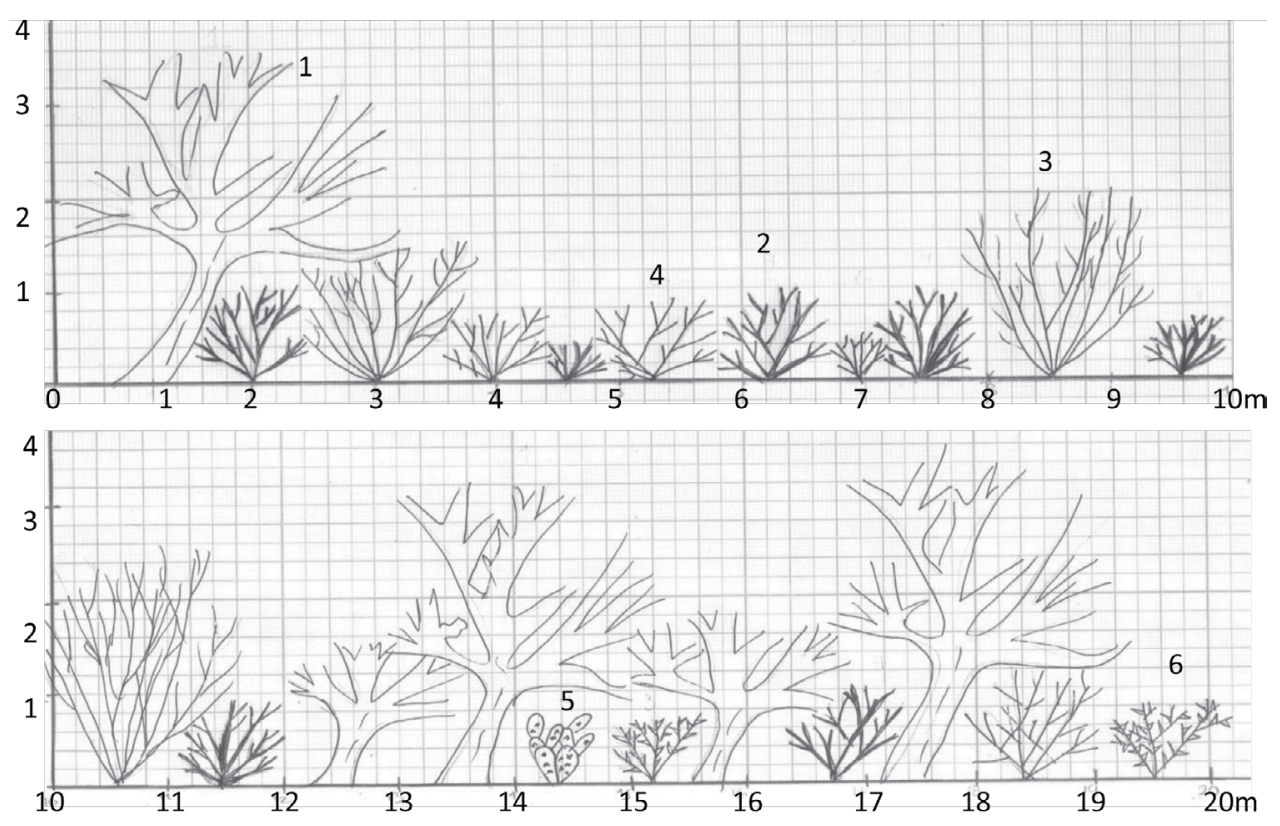

Figura 5. Perfil estructural vertical de la vegetación dominada por árboles en un matorral xerófilo en el Valle del Mezquital, Hidalgo. 1 Prosopis laevigata, 2 Flourensia resinosa, 3 Karwinskia humboldtiana, 4 Mimosa depauperata, 5 Opuntia streptacantha, 6 Condalia mexicana. 


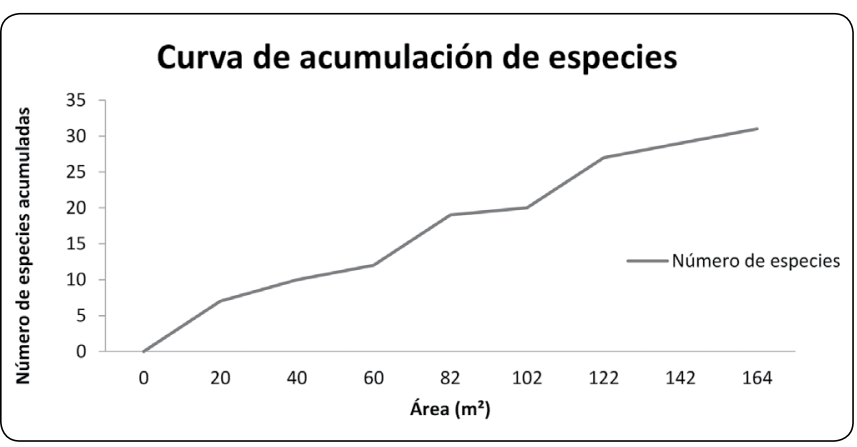

Figura 6. Curva de acumulación de especies vegetales observadas en un matorral xerófilo en el Valle del Mezquital, Hidalgo.

la acumulación de las especies vegetales muestreadas en campo y la Figura 7 (datos calculados), es una curva de rarefacción donde se muestra el esfuerzo de muestreo; ambas su obtuvieron para la vegetación del matorral xerófilo analizado.

Índice de Valor de Importancia. Las especies con altos IVI presentes en las ocho UM fueron: Mimosa depauperata, Mimosa biuncifera y Prosopis laevigata. En el Cuadro 2, se presentan los IVI más elevados de las especies muestreadas.

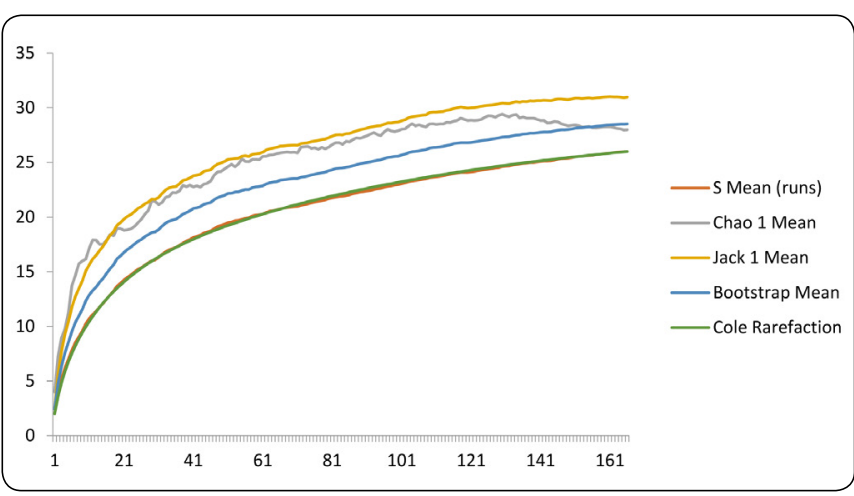

Figura 7. Curvas de acumulación de especies de la riqueza observada y la riqueza estimada por cinco estimadores no paramétricos, para el matorral xerófilo del Valle del Mezquital, Hidalgo.

Para las etapas serales dominados por: herbáceas perennes, arbustos bajos, arbustos medianos y árboles, el valor de significancia obtenido fue de $\mathrm{p}=0.8453$, por lo que no hay diferencias significativas entre los IVI de las etapas serales (Figura 8).

\begin{tabular}{|c|c|c|c|c|c|}
\hline & Especie característica & $\begin{array}{c}\text { Buchlö̈ } \\
\text { dactyloides }\end{array}$ & $\begin{array}{c}\text { Mimosa } \\
\text { depauperata }\end{array}$ & Mimosa biuncifera & $\begin{array}{l}\text { Prosopis } \\
\text { laevigata }\end{array}$ \\
\hline Familia & $\begin{array}{r}\text { Forma de vida: } \\
\text { Especie (tipo de planta) }\end{array}$ & $\begin{array}{c}\text { Herbáceas } \\
\text { perennes }\end{array}$ & $\begin{array}{c}\text { Arbustos bajos } \\
\quad(<1 \mathrm{~m})\end{array}$ & $\begin{array}{l}\text { Arbustos medianos } \\
\qquad(>1 \mathrm{~m})\end{array}$ & $\begin{array}{c}\text { Árboles } \\
\text { (un solo fuste) }\end{array}$ \\
\hline Agavaceae & $\begin{array}{l}\text { Agave salmiana var. Salmiana Otto ex Salm- } \\
\text { Dyck (leñosa rosetófila) }\end{array}$ & 7.66 & 30.96 & 16.3 & 15.29 \\
\hline Asteraceae & $\begin{array}{l}\begin{array}{l}\text { Flourensia resinosa (Brandegee) S.F. Blake } \\
\text { (arbusto medio) }\end{array} \\
\text { (a) }\end{array}$ & 57.78 & 48.58 & 42.48 & 65.13 \\
\hline Poaceae & $\begin{array}{l}\text { Buchloë dactyloides (Nutt.) Engelm. } \\
\text { (gramínea) }\end{array}$ & 77.82 & 39.05 & 44.41 & 0 \\
\hline Rhamnaceae & Condalia mexicana Schlecht. (arbusto medio) & 9.8 & 13.79 & 24.66 & 15.71 \\
\hline Rhamnaceae & $\begin{array}{l}\text { Karwinskia humboldtiana (Romer \& } \\
\text { Schultes) Zucc. (arbusto medio) }\end{array}$ & 0 & 0 & 0 & 30.6 \\
\hline Fabaceae & Mimosa biuncifera Benth. (arbusto medio) & 11.29 & 0 & 72.33 & 0 \\
\hline Fabaceae & Mimosa depauperata Benth. (arbusto bajo) & 103.49 & 98.02 & 22.92 & 34.1 \\
\hline Fabaceae & $\begin{array}{l}\text { Prosopis laevigata (Humb. \& Bonpl. ex } \\
\text { Willd.) M. C. Johnst. (arbustiva o arbórea) }\end{array}$ & 0 & 0 & 19.77 & 85.95 \\
\hline Cactaceae & $\begin{array}{l}\text { Opuntia cantabrigiensis Lynch, } 1903 . \\
\text { (crasicaule) }\end{array}$ & 7 & 15.57 & 16.7 & 0 \\
\hline Cactaceae & Opuntia streptacantha Engelm. (crasicaule) & 0 & 0 & 0 & 10.23 \\
\hline Cactaceae & Opuntia imbricata (Haw.) DC. (crasicaule) & 0 & 13.55 & 12.56 & 8.07 \\
\hline
\end{tabular}

Cuadro 2. Índice de Valor de Importancia (IVI) de las especies con los valores más elevados, en cuatro etapas de desarrollo de un matorral xerófilo en el Valle del Mezquital, Hidalgo. En negritas los valores más altos de cada mosaico vegetal, según la especie característica. 
Índice de diversidad. En la Figura 9 se muestra la diversidad (índice de Shannon-Wiener) para la vegetación en cuatro etapas serales de un matorral xerófilo. La prueba de análisis de varianza aplicada para el índice de Shannon-Wiener indicó que no hay una diferencia significativa para este parámetro entre los mosaicos vegetales analizados $(p=0.2667)$. En relación con la diversidad de HMA (Figura 10), se presenta el índice de diversidad Shannon-Wiener $\left(\mathrm{H}^{\prime}\right)$ para las morfoespecies de HMA en las cuatro etapas serales de un matorral xerófilo, sin registrar diferencias estadísticas entre etapas serales. Los valores obtenidos de este índice para la vegetación y para los HMA fueron bajos (cercanos a 2), de acuerdo con la clasificación de Margalef (1972), lo cual corresponde a tipos de vegetación de ambientes semiáridos.

Determinación y extracción de esporas. El mosaico vegetal que tuvo el mayor número de morfoespecies (15 spp.) fue el

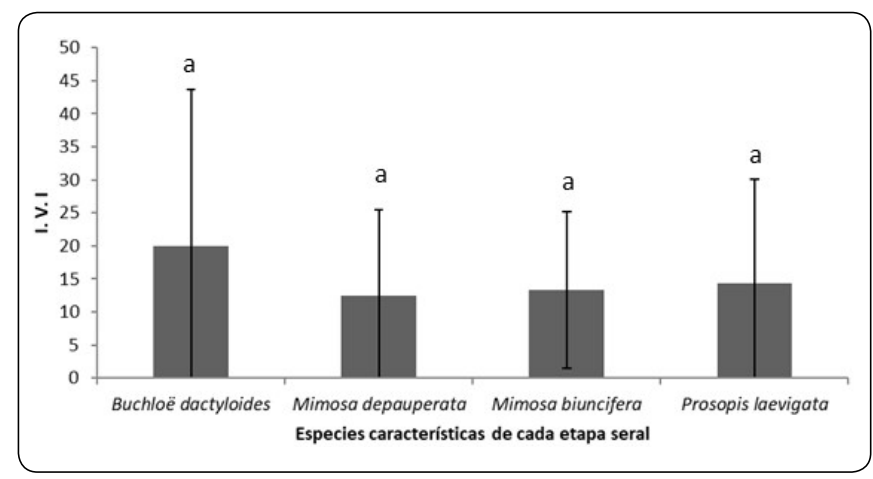

Figura 8. Índice de Valor de Importancia (IVI) de cuatro mosaicos vegetales de un matorral xerófilo. Los histogramas representan el valor promedio para cada etapa seral y las barras una desviación estándar. Las letras minúsculas iguales sobre los histogramas indican que no hay diferencias estadísticas significativas $(p<0.05)$.

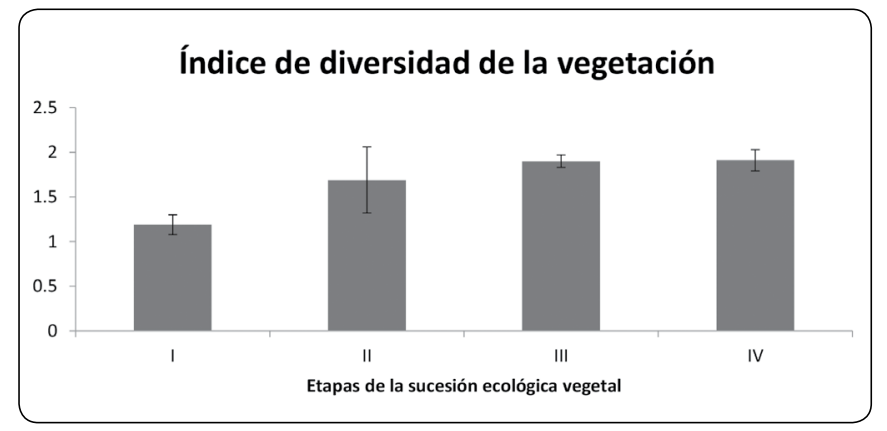

Figura 9. Índice de diversidad de Shannon-Wiener (H') de la vegetación en un matorral xerófilo, en el Valle del Mezquital, Hidalgo. Las barras representan una desviación estándar por arriba y por debajo de la media. I. Especies pioneras, II Especies intermedias tempranas, III. Especies intermedias tardías, IV. Especies maduras. Estadísticamente no se encontraron diferencias significativas entre los 4 mosaicos vegetales $(p<0.05)$.

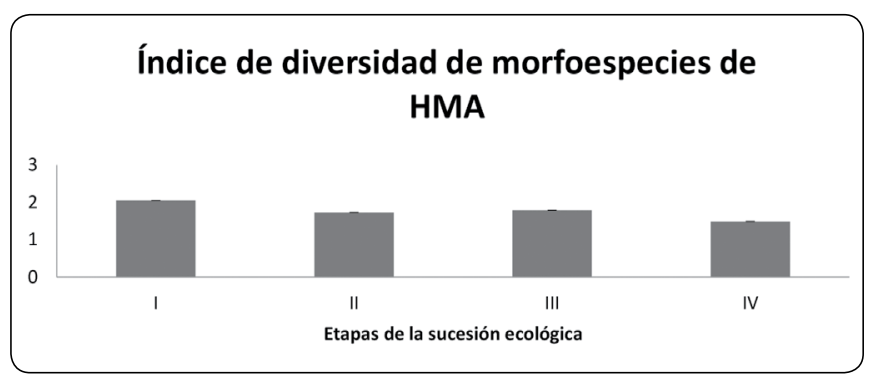

Figura 10. Índice de diversidad Shannon-Wiener de morfoespecies de hongos micorrizógenos arbusculares (HMA) en un matorral xerófilo, en el Valle del Mezquital, Hidalgo. I. Especies pioneras, II Especies intermedias tempranas, III. Especies intermedias tardías, IV. Especies maduras. Estadísticamente no se encontraron diferencias significativas $(p<0.05)$.

de Mimosa biuncifera, mientras que el menor número (13 spp.) correspondió al de Mimosa depauperata y Prosopis laevigata. El género representado por un mayor número de morfoespecies fue Glomus, seguido de Acaulospora y Funneliformis (Cuadro 3 y Figura 11). En la abundancia de esporas el género Glomus fue el más representativo, seguido de Acaulospora.

Porcentaje de colonización micorrízica. La Figura 12 muestra el porcentaje de colonización micorrízica de hifas, vesículas y arbúsculos de raíces de plantas de Buchloë dactyloides, y de gramíneas asociadas a Mimosa depauperata, Mimosa biuncifera y Prosopis laevigata. La colonización micorrízica total (Figura 13), muestra que las gramíneas asociadas a los mosaicos con dominancia de Mimosa depauperata fueron los que presentaron una colonización mayor (88\%), seguidos de Prosopis laevigata (86\%) y de Mimosa biuncifera (85\%), mientras que el mosaico caracterizado por Buchloë dactyloides fue el de menor porcentaje (67\%). En los registros gráficos de la colonización micorrízica, las hifas y vesículas se encontraron en mayores cantidades, mientras que los arbúsculos estuvieron casi ausentes, a excepción de las raíces de las gramíneas presentes en los mosaicos dominados por Mimosa depauperata y Mimosa biuncifera.

Distribución de la Abundancia Relativa. La curva de la distribución de la abundancia relativa (RAD por sus siglas en inglés: relative abundance distribution) de las especies de una comunidad, muestra la distribución de la dominanciacomposición de un conjunto de especies, es decir, la estructura general de las abundancias específicas de sus componentes. Así, las Figuras 14-17 presentan las curvas RAD de las especies vegetales y de las morfoespecies de HMA, para las cuatro etapas serales, las cuales están caracterizadas, en el orden de maduración de la comunidad vegetal, por: Buchloë dactyloides, Mimosa depauperata, Mimosa biuncifera y Prosopis laevigata. Los resultados obtenidos mediante la prueba de chi cuadrada 


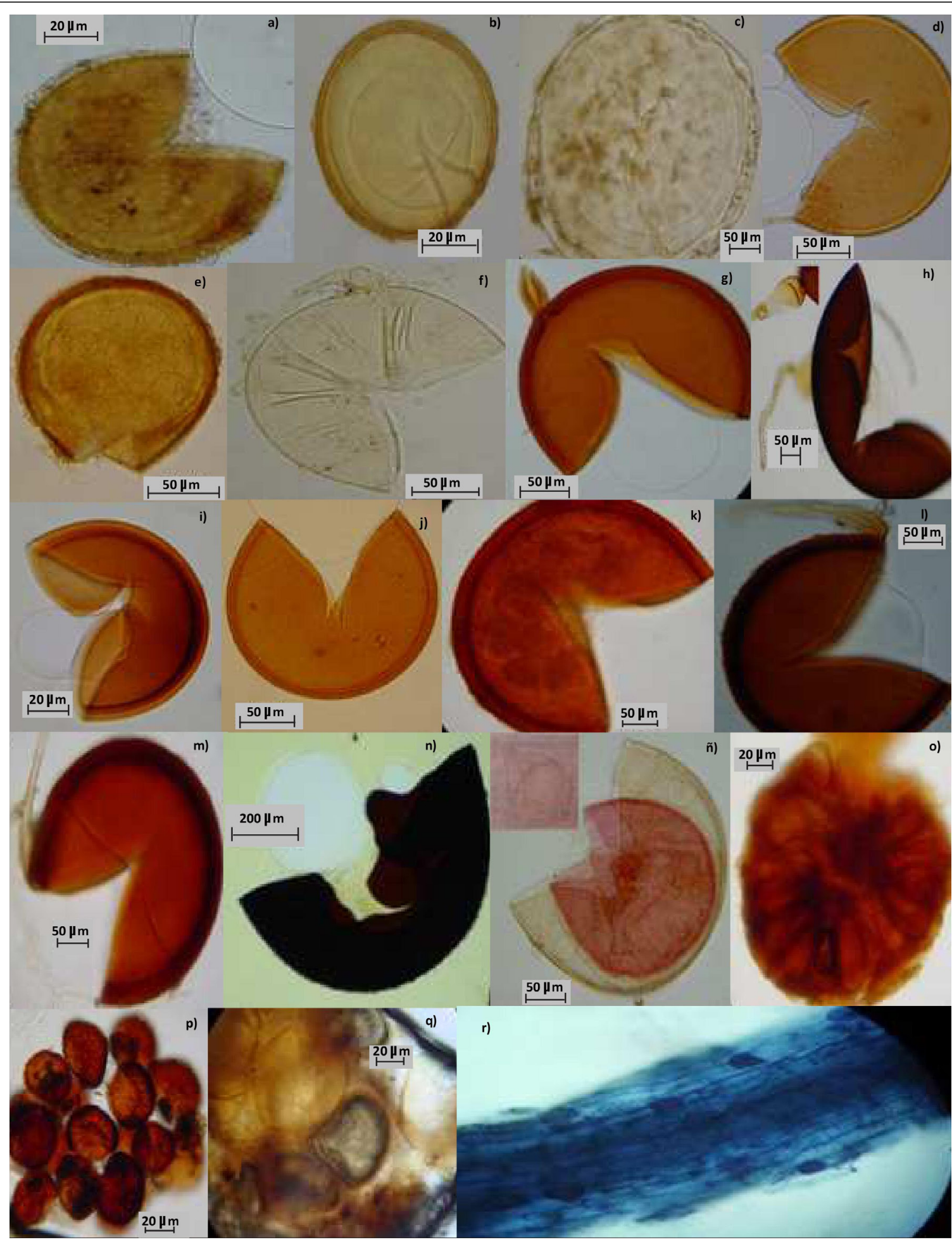

Figura 11. a) Acaulospora denticulata detalle de la ornamentación; b) Acaulospora sp. c) Diversispora sp. d) Claroideoglomus sp. e) Entrophospora infrequens con detalles de la ornamentación. f) Funneliformis mosseae con hifa en forma de embudo. g) Funneliformis geosporum con hifa en forma de embudo delgado y capa laminar gruesa. h) Gigaspora sp. y en el recuadro superior izquierdo se muestra la hifa bulbosa. i) Glomus caesaris; j) Glomus sp. 1. k) Glomus sp. 2. I) Glomus sp. 3. m) Glomus sp. 5. n) Racocetra gregaria. ñ) Scutellospora sp. y en el recuadro superior izquierdo se observa la hifa en forma de bulbo con reacción al reactivo Melzer de la capa interna. o) Sclerocystis sinuosa. p) Sclerocystis sp. 1. q) Sclerocystis sp. 2. r) Colonización micorrízica en raíz de Buchloë dactyloides, donde se aprecian hifas y vesículas. 


\begin{tabular}{|c|c|c|c|c|}
\hline \multirow[b]{2}{*}{ Morfoespecies } & \multicolumn{4}{|c|}{ Especie característica } \\
\hline & $\begin{array}{c}\text { Buchlö̈ } \\
\text { dactyloides }\end{array}$ & $\begin{array}{c}\text { Mimosa } \\
\text { depauperata }\end{array}$ & $\begin{array}{c}\text { Mimosa } \\
\text { biuncifera }\end{array}$ & $\begin{array}{l}\text { Prosopis } \\
\text { laevigata }\end{array}$ \\
\hline Acaulospora aff. denticulata & $\mathrm{X}$ & $\mathrm{X}$ & $\mathrm{X}$ & \\
\hline Acaulospora sp. & $\mathrm{X}$ & $\mathrm{X}$ & $\mathrm{X}$ & $\mathrm{X}$ \\
\hline Diversispora sp. & & $\mathrm{X}$ & $\mathrm{X}$ & $\mathrm{X}$ \\
\hline Claroideoglomus sp. & & $\mathrm{X}$ & & $\mathrm{X}$ \\
\hline $\begin{array}{l}\text { Entrophosphora infrecuens (I.R. Hall) R.N. Ames } \\
\text { \& R.W. Schneid. }\end{array}$ & $X$ & & & \\
\hline $\begin{array}{l}\text { Funneliformis mosseae (T.H. Nicolson \& Gerd.) C. } \\
\text { Walker \& A. Schüßler }\end{array}$ & $X$ & $X$ & $X$ & $X$ \\
\hline $\begin{array}{l}\text { Funneliformis geosporum (T.H. Nicolson \& Gerd.) } \\
\text { C. Walker \& A. Schüßler }\end{array}$ & $\mathrm{X}$ & $\mathrm{X}$ & $\mathrm{X}$ & $\mathrm{X}$ \\
\hline Gigaspora sp. & & & $\mathrm{X}$ & \\
\hline Glomus caesaris Sieverd. \& Oehl & $\mathrm{X}$ & $\mathrm{X}$ & $\mathrm{X}$ & $\mathrm{X}$ \\
\hline Glomus sp. 1 & $\mathrm{X}$ & $\mathrm{X}$ & $\mathrm{X}$ & $\mathrm{X}$ \\
\hline Glomus sp. 2 & $\mathrm{X}$ & $\mathrm{X}$ & $\mathrm{X}$ & $\mathrm{X}$ \\
\hline Glomus sp. 3 & $\mathrm{X}$ & $\mathrm{X}$ & $\mathrm{X}$ & $\mathrm{X}$ \\
\hline Glomus sp. 4 & $\mathrm{X}$ & & $\mathrm{X}$ & $\mathrm{X}$ \\
\hline Glomus sp. 5 & $\mathrm{X}$ & $\mathrm{X}$ & $\mathrm{X}$ & $\mathrm{X}$ \\
\hline Racocetra gregaria & & $\mathrm{X}$ & $\mathrm{X}$ & \\
\hline Scutellospora sp. & & $\mathrm{X}$ & $\mathrm{X}$ & \\
\hline Sclerocystis sinuosa Gerd. \& B.K. Baksh & $\mathrm{X}$ & & $\mathrm{X}$ & $\mathrm{X}$ \\
\hline Sclerocystis sp. 1 & $\mathrm{X}$ & & & \\
\hline Sclerocystis sp. 2 & & $\mathrm{X}$ & & $\mathrm{X}$ \\
\hline TOTAL & 13 & 14 & 15 & 13 \\
\hline
\end{tabular}

Cuadro 3. Morfoespecies de hongos micorrizógenos arbusculares en cuatro etapas serales de la vegetación de un matorral xerófilo del Valle del Mezquital, Hidalgo.

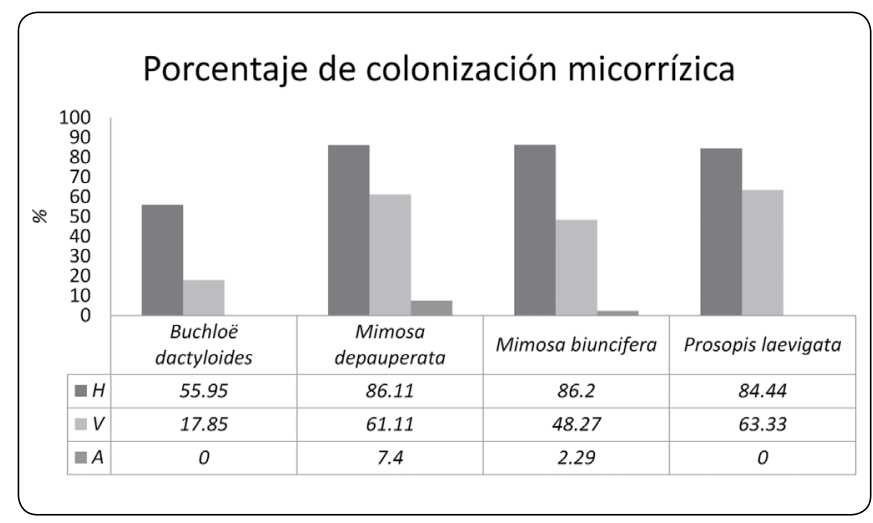

Figura 12. Porcentaje de colonización de hifas $(\mathrm{H})$, vesículas (V) y arbúsculos (A) en raíces de Buchloë dactyloides y de gramíneas bajo la cobertura de Mimosa depauperata, Mimosa biuncifera y Prosopis laevigata.

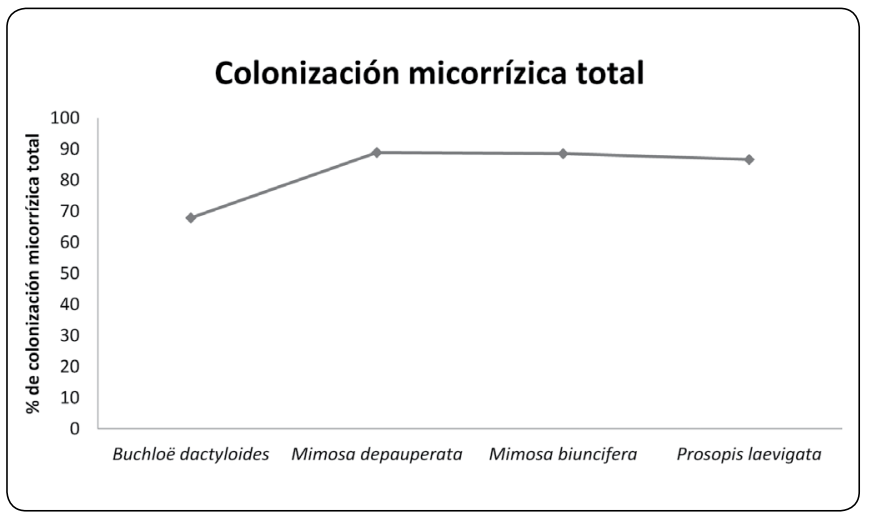

Figura 13. Porcentaje de colonización micorrízica total (CT) en raíces de Buchloë dactyloides, y de gramíneas bajo la cobertura de Mimosa depauperata, Mimosa biuncifera y Prosopis laevigata. 


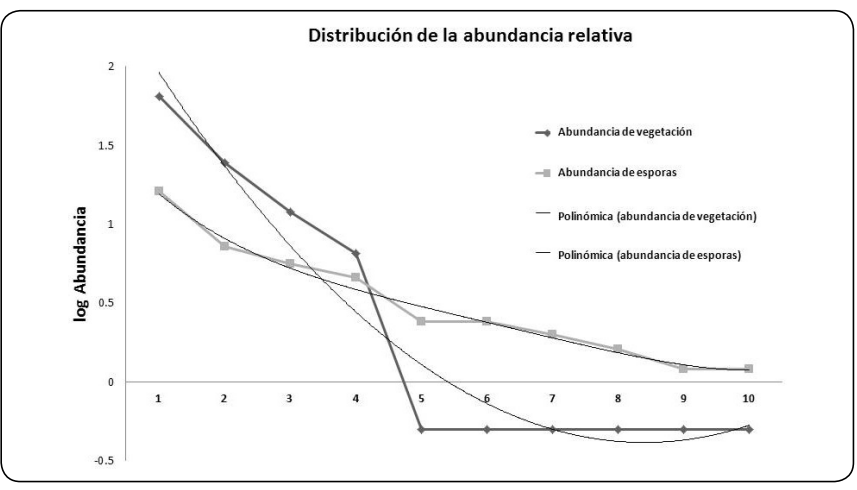

Figura 14. Distribución de la abundancia relativa entre morfoespecies de HMA y vegetación, donde una prueba de $X_{i}^{2}$ mostró un ajuste estadísticamente significativo $(p<0.05)$ entre curvas, en la etapa seral caracterizada por Buchloë dactyloides.

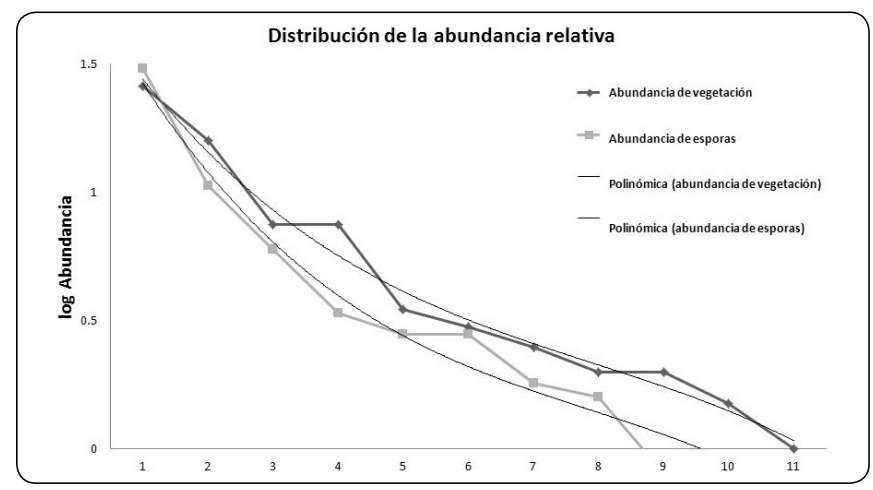

Figura 16. Distribución de la abundancia relativa entre morfoespecies de HMA y vegetación, donde una prueba de $\mathrm{X}_{i}^{2}$ mostró un ajuste estadísticamente significativo $(\mathrm{p}<0.05)$ entre curvas, en la etapa seral dominada por Mimosa biuncifera.

$\left(\chi_{i}^{2}\right)$ de bondad de ajuste entre las curvas, $\operatorname{con} \alpha=0.05$, muestran un mismo tipo de estructura de las comunidades de plantas y de morfoespecies de HMA, por lo que sí hay una relación de composición y dominancia entre los HMA y la vegetación en las cuatro etapas serales: Buchloe dactyloides $\left(\chi_{i}^{2}=7.98\right.$, $\mathrm{gl}=16.91)$, Mimosa depauperata $\left(\chi_{i}^{2}=3.04 \mathrm{gl}=23.68\right)$, Mimosa biuncifera $\left(\chi_{i}^{2}=1.00, \mathrm{gl}=23.68\right)$ y Prosopis laevigata $\left(\chi_{i}^{2}=\right.$ 2.27, $\mathrm{gl}=23.68)$.

\section{Discusión}

Perfil vertical. En los matorrales xerófilos, el eje conductor del desarrollo de la vegetación es la disponibilidad hídrica del suelo para el crecimiento vegetal y son las comunidades de HMA, mediante la malla hifal, las que facilitan este recurso a las comunidades vegetales para que lleven a cabo sus procesos funcionales (Miranda, 1955). Asimismo, al ser el factor hídrico un limitante para la productividad vegetal, tanto la densidad

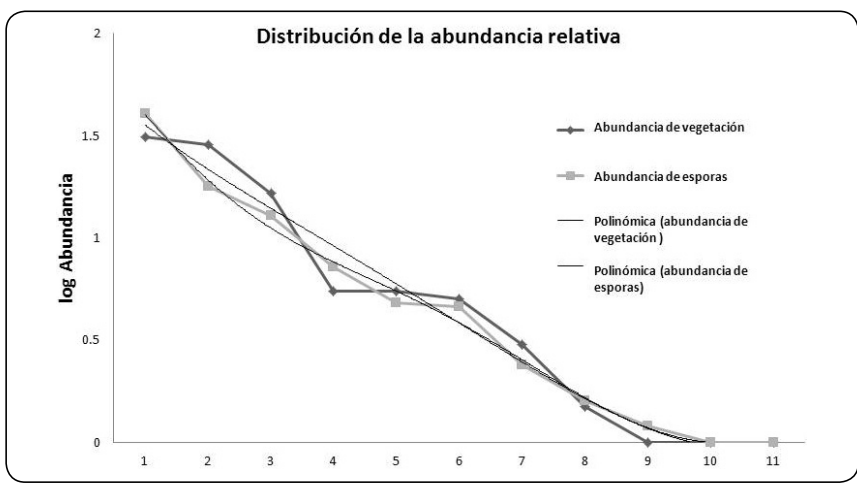

Figura 15. Distribución de la abundancia relativa entre morfoespecies de HMA y vegetación, donde una prueba de $X_{i}^{2}$ mostró un ajuste estadísticamente significativo $(p<0.05)$ entre curvas, en la etapa seral dominada por Mimosa depauperata.

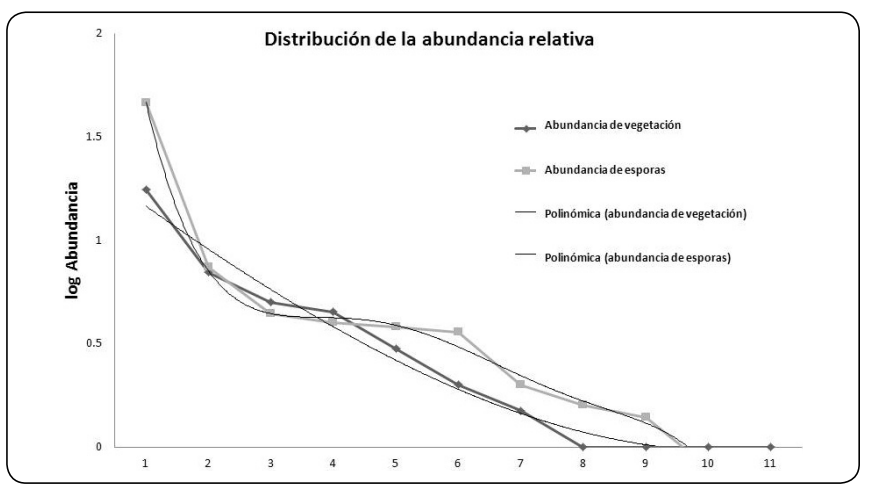

Figura. 17. Distribución de la abundancia relativa entre morfoespecies de HMA y vegetación, donde una prueba de $X_{i}^{2}$ mostró un ajuste estadísticamente significativo $(p<0.05)$ entre curvas, en la etapa seral dominada por Prosopis laevigata.

como la composición de especies de plantas influyen en los procesos de formación del suelo y en el hábitat de los HMA (Huerta-Martínez \& García-Moya, 2004). Así, la interacción mutualista y dinámica entre fitobiontes y micobiontes se manifiesta durante el establecimiento, desarrollo y maduración de ambas comunidades. Seguramente, las plantas dominantes influyen en la composición y distribución de los individuos en los mosaicos vegetales durante la sucesión ecológica. Esta influencia puede darse de forma similar al papel organizador de los gremios vegetales que realizan los denominados "árboles madre" (Monroy-Ata, Peña-Becerril, \& García-Díaz, 2016), los cuales propagan la inoculación micorrízica entre plantas vecinas mediante esporocarpos o la red hifal, determinando la estructura y diversidad de los mosaicos.

Curva de acumulación de especies. En este trabajo se registraron 31 especies vegetales, lo que puede indicar una riqueza baja en 
comparación con los estudios de Villavicencio, Pérez \& Ramírez (1998) en los matorrales xerófilos de Hidalgo, en los cuales han reportado la presencia de 70 especies arbustivas o en otras comunidades similares como las estudiadas en Querétaro por Treviño \& Hernández (2000), quienes reportaron 83 especies. Asimismo, las de Coahuila por Encina-Domínguez, Meave \& Zárate-Lupercio (2013) que registraron 97 especies o las descritas en Tamaulipas por Alanís-Rodríguez et al. (2015) quienes reportaron 35 especies. Esto se puede explicar por ser un matorral xerófilo con signos de perturbación por sobrepastoreo, la menor superficie de muestreo y que éste se realizó sólo en la época seca del año. La familia mejor representada fue Fabaceae, con cuatro géneros. La curva de rarefacción calculada para estimar la riqueza de especies local mostró una eficiencia de muestreo del 93\%; seguramente las plantas no registradas corresponden a especies palatables para el ganado, como las gramíneas. Esto permite explicar la relativamente baja diversidad vegetal del hábitat muestreado (Sonco, 2013).

Índice de Valor de Importancia. Las especies con altos IVI registrados en los mosaicos vegetales analizados fueron: Mimosa depauperata, Mimosa biuncifera y Prosopis laevigata, mientras que Buchloë dactyloides fue representativo sin ser un valor alto. Campo \& Duval (2014) mencionan que aquellas especies que presentan los mayores valores son las que controlan en mayor medida el flujo de energía (mediante su cobertura vegetal, en este caso) y frecuentemente presentan un patrón regular es su distribución; no obstante, aquéllas con valor bajo son especies no dominantes, de distribución dispersa o raras. En efecto, en los cuatro mosaicos vegetales muestreados se presentó un patrón regular en cuanto a distribución y abundancia de las especies dominantes, al determinar las curvas de distribución de abundancias relativas. Este patrón consistió en una especie dominante, con otras plantas asociadas y algunas especies poco frecuentes.

Índice de diversidad. La mayor diversidad se registró en el mosaico vegetal dominado por Prosopis laevigata (1.91), comparable con el índice de diversidad (H') de 2.5, obtenido en los resultados de un trabajo realizado por Montaño-Arias, García-Sánchez, Ochoa-de la Rosa \& Monroy-Ata (2006), en el municipio de Santiago de Anaya, Hidalgo, sobre la vegetación dominada por Prosopis laevigata; de igual manera, García-Sánchez (2011) reportó que el sitio muestreado con una codominancia de Mimosa biuncifera y Prosopis laevigata, en el Valle del Mezquital, Hidalgo, obtuvo la mayor diversidad de especies en comparación con sitios donde cada leguminosa era la dominante; Huerta \& García (2004), en otros estudios sobre cuatro distintos tipos de matorrales xerófilos, registraron valores de H' de 1.07 a 2.17. También, Alanís-Rodríguez et al. (2015), reportaron valores entre 0.36 y 1.36 para matorrales rosetófilos en el noreste de México. Por otro lado, en trabajos realizados en una comunidad madura de matorral espinoso tamaulipeco se reportan valores entre 1.94 y 2.40 (Mora et al., 2013; Ramírez-Lozano et al., 2013; González-Rodríguez, Ramírez-Lozano, Cantú-Silva, Gómez-Meza \& Uvalle-Sauceda, 2010; Treviño \& Hernández, 2000). Adicionalmente, Mata et al. (2014) evaluaron comunidades maduras de tres matorrales del noreste de México (matorral desértico micrófilo, matorral desértico rosetófilo y matorral submontano) y obtuvieron valores de $1.90,1.89$ y 1.88 respectivamente, por lo que los valores de H' obtenidos en este trabajo están dentro de un rango similar en el índice de diversidad para matorrales xerófilos en México, lo que indica que los mosaicos analizados pueden ser comparables a otros gremios vegetales de ambientes semiáridos.

Para la diversidad de HMA el valor más alto se obtuvo en el mosaico vegetal caracterizado por Buchloë dactyloides (2.05). Asimismo, en trabajos relacionados con morfoespecies de HMA, García-Sánchez (2011), en el Valle del Mezquital, Hidalgo, obtuvo el valor del índice de diversidad de HMA asociados a Mimosa biuncifera y a Prosopis laevigata, encontrando valores altos para este índice. También, Muñoz (2013) realizó un estudio para seis especies vegetales provenientes del Municipio de Santiago de Anaya, Hidalgo, donde evaluó el índice de diversidad de Shannon-Wiener para las comunidades de esporas y obtuvo un valor máximo de 1.29 para Agave lechuguilla. Estos resultados sugieren que una etapa seral temprana tiene alta diversidad de micobiontes, debido a que la asociación HMA-vegetación incrementa la tasa de supervivencia y el establecimiento de plantas.

Determinación y extracción de esporas. Los géneros mejor representados para el matorral xerófilo fueron Glomus, seguido de Acaulospora y Funneliformis. En estudios realizados por Medrano (2002) menciona que el género Glomus es el más abundante en suelos de matorrales xerófilos de Santiago de Anaya, Hidalgo y probablemente es el que presenta más resistencia a la sequía edáfica y por lo tanto es una especie con alta funcionalidad bajo las condiciones semiáridas del sitio de estudio (Montaño-Arias, 2000). Algunas especies del género Glomus son de amplia distribución en ambientes áridos y semiáridos, pues se han encontrado en casi todos los hábitats asociadas a una amplia gama de especies vegetales. En el trabajo realizado por Montaño-Arias (2000) también se reporta la presencia de los géneros Glomus y Scutellospora y señala a Glomus como el más representado para el sitio. Asimismo, García-Sánchez (2011) reportó para el Valle del Mezquital, Hidalgo, el 40\% de las especies de HMA pertenecientes a la familia Glomeraceae. También, Chimal-Sánchez,Araiza-Jacinto \& Román-Cárdenas (2015), en un estudio realizado en el Parque Ecológico "Cubitos", reportaron que el 54.5\% de la riqueza de HMA pertenece a los géneros Claroideoglomus, Funneliformis y Glomus (Glomerales) y que en la rizosfera de Mimosa biuncifera se encontró la mayor riqueza de micobiontes, con seis especies, en un sitio conservado. En otro estudio realizado por Chimal-Sánchez, García-Sánchez \& Hernández-Cuevas (2015) en el Valle del Mezquital, Hidalgo, encontraron una riqueza 
de especies de HMA de 29 morfo-especies y las mayormente representadas fueron Glomeraceae y Gigasporaceae (aportando el 62\% de las especies); de la misma manera, Hernández (2011) realizó un estudio para Mimosa biuncifera en un matorral xerófilo del Valle del Mezquital, Hidalgo y los resultados que obtuvo para la densidad de esporas en una zona perturbada y una conservada es de 341 esporas y 114 respectivamente; asimismo, obtuvo 15 morfoespecies de HMA para la zona perturbada y 21 morfoespecies para la zona conservada. Por otra parte, en un estudio realizado en un matorral xerófilo en el Valle de Actopan, Hidalgo, Monroy-Ata, Estevez-Torres, García-Sánchez \& Ríos (2007) encontraron una dominancia del género Glomus en suelo circundante a raíces del zacate navajita azul (Bouteloua gracilis (Willd. ex Kunth) Lag. ex Griffiths), mencionando que posiblemente el papel de las comunidades de hongos micorrízicos con dominancia de Glomus, en etapas serales tempranas, sea promover el establecimiento y crecimiento de las plantas hospederas. Por otro lado, Camargo-Ricalde \& Esperón-Rodríguez (2005) estudiaron la importancia de las islas de recursos formadas por especies de Mimosa en el Valle semiárido de Tehuacán-Cuicatlán como reservorios de esporas de HMA y encontraron la dominancia de los géneros Glomus y Acaulospora. También, se ha reportado que cuando un suelo se encuentra deteriorado hay un aumento en las esporas más pequeñas, generalmente de la familia Glomeraceae y del género Acaulospora (Allen, Allen, Egerton-Warburton, Corkidi \& Gómez-Pompa, 2003), ya que parecen ser más tolerantes a las perturbaciones del suelo, en comparación con las esporas de gran tamaño de la familia Gigasporacea (Boddington \& Dodd, 2000). La dominancia del género Glomus en ambientes perturbados y en estadios sucesionales tempranos puede deberse a sus elevadas tasas de esporulación(Husband, Herre, Turner, Gallery \& Young, 2002; Bever, Morton, Antonovics \& Schultz, 1996), ya que es un mejor promotor del crecimiento de plántulas con respecto a Gigaspora y Scutellospora, cuyas especies son colonizadoras más lentas, que normalmente producen un balance negativo de carbono y fósforo en etapas sucesionales tempranas y en hospederos jóvenes, pero que promueven el desarrollo de la red hifal (Allen et al, 2003). Así, los resultados obtenidos en este trabajo se relacionan con datos reportados en otros matorrales xerófilos de México, ya que se registró que en las etapas serales tempranas a intermedias se encontraron los géneros Glomus y Acaulospora como dominantes, lo que puede indicar cierto nivel de deterioro en suelo y vegetación de la zona de estudio; también, se muestra una relación entre las esporas de Gigaspora y Scutellospora con las etapas serales intermedias a tardías, lo que puede indicar su papel en la formación de la red hifal durante el proceso de maduración de la comunidad biótica.

En este trabajo se registró, en la etapa seral dominada por Prosopis laevigata, una abundancia máxima de 45 esporas por $100 \mathrm{~g}$ de suelo seco y una riqueza de HMAde 13 morfoespecies. De los trabajos realizados para Prosopis laevigata, Monroy-Ata, Peña-Becerril \& García-Díaz(2016) obtuvieron una abundancia de esporas con un máximo de 206 y un mínimo de 75 por $100 \mathrm{~g}$ de suelo seco y para la riqueza de HMA reportaron seis géneros. Asimismo, García-Sánchez et al. (2012), en un estudio realizado en el Valle del Mezquital, Hidalgo, encontraron una abundancia de esporas de HMA mayor bajo la copa de Prosopis laevigata y Mimosa biuncifera.

Porcentaje de colonización micorrízica. Los porcentajes de colonización obtenidos para gramíneas asociadas y para Buchlö̈ dactyloides fueron altos $(88 \%, 86 \%, 85 \%$ y 67\%). Miller, Reinhardt \& Jastrow (1995) reportan que en temporada de secas el micelio permanece principalmente por la presencia de plantas crasas (cactáceas, agaváceas y crasuláceas) y seguramente, por el aporte invernal de agua mediante el rocío matinal, mientras que la producción de raíces se reduce; por ello, el micelio puede facilitar las respuestas funcionales de desarrollo vegetal en condiciones de disponibilidad hídrica limitada en el perfil edáfico. Por ello y en el caso de la colonización radical por arbúsculos reportada en este estudio, su presencia puede deberse a que es la estructura donde ocurre el mayor intercambio de metabolitos entre plantas y HMA y en la temporada de secas las plantas están limitadas no sólo por el estrés hídrico, sino por una baja disponibilidad de nutrimentos como el nitrógeno y el fósforo. Barragán (2003) menciona que el porcentaje de colonización micorrízica indica el nivel de interacción entre fito y micobiontes, de tal manera que entre más alto sea el porcentaje de colonización fúngica, mayor influencia tendrá en el crecimiento y desarrollo de una planta; también reportó ( $o p$. cit.) un porcentaje máximo de $80 \%$ en plantas inoculadas en invernadero, lo cual es un porcentaje menor a los porcentajes de colonización micorrízica obtenidos en este trabajo para condiciones de campo. Esto significa, posiblemente, que los vegetales con elevados niveles de micorrización, mitigan el estrés hídrico y aumentan su crecimiento y vigor, según lo que reportan Rubio, Cepeda, Borie \& Contreras (1997). El porcentaje de colonización micorrízica para Mimosa biuncifera fue del $88.5 \%$; este valor podría estar relacionado con lo reportado por Cruz (2013), quien menciona que el micelio es importante para la funcionalidad de las plantas bajo condiciones de estrés hídrico, como lo han reportado estudios en los que la producción de micelio aumentó cuando hay baja disponibilidad de agua para las plantas (Khalvati, Hu, Mozafar \& Schmidhalter, 2005; Bethlenfalvay, Brown, Ames \& Thomas, 1998; Davies, Potter \& Linderman, 1992). Este resultado es similar al obtenido por Hernández (2011), quien registró una colonización micorrízica para $M$. biuncifera de $70 \%$, con un inóculo proveniente de una zona perturbada y $55 \%$ con inóculo de una zona conservada; asimismo, este valor se encuentra dentro del rango obtenido por Muñoz (2013), quien calculó porcentajes de colonización total micorrízica para diferentes especies vegetales de un matorral xerófilo, inoculadas en invernadero, comprendidos entre 80 y 94.9\%. También, García-Sánchez, Monroy-Ata \& Chimal-Sánchez (2007) reportaron una colonización del 54\% en Mimosa depauperata. Por otra parte, para Prosopis laevigata 
se ha registrado una colonización micorrízica que varía entre 3.5\% y 53\% (Monroy-Ata, Peña-Becerril \& García-Díaz, 2016; García-Sánchez, Monroy-Ata \& Chimal-Sánchez, 2007).

Distribución de la Abundancia Relativa. El modelo logarítmico de la abundancia relativa de las especies, agrupadas por rangos de frecuencia, muestra una dominancia de especies vegetales con mayor presencia por densidad o cobertura, lo que refleja un mayor control del flujo de energía solar, en las cuatro etapas serales analizadas. Para las comunidades de morfoespecies de HMA asociados a los cuatro mosaicos vegetales, se obtuvieron patrones de composición y abundancia semejantes a los de las plantas, ya que la silueta de las curvas es en forma de "S invertida", aproximadamente, en ambos casos y para las cuatro etapas serales. Esto refleja una correspondencia entre ambas comunidades con especies dominantes y que tanto los fitobiontes como los micobiontes se encuentran vinculados mediante estructuras similares en distintos niveles de organización, ya que la forma de la curva de la distribución de abundancias relativas muestra la organización estructural, en composición y abundancia de especies, de una comunidad vegetal(Wilson,1999). Asimismo, Wilson(1999) menciona que el crecimiento de las plantas se ve afectado por otras especies vegetales con las que coexisten y por varios factores ambientales. Además, Monroy-Ata, Peña-Becerril \& García-Díaz (2016) reportan que la estructura de la comunidad vegetal refleja la dinámica edáfica del agua, debido a los pulsos de precipitación y evapotranspiración, y a la distribución espacio-temporal del potencial hídrico del suelo, lo que explicaría la dominancia de especies vegetales denominadas nodriza (Monroy-Ata, Peña-Becerril \& García-Díaz, 2016; Miller \& Jastrow, 1992) en matorrales xerófilos y su estrecha relación con la red hifal de los HMA.

\section{Conclusiones}

En este estudio se mostró que hay una correspondencia entre el patrón de dominancias específicas de las especies vegetales y el de las comunidades de morfo-especies de HMA, de cada una de las cuatro etapas serales de un matorral xerófilo. Las fases sucesionales analizadas fueron las siguientes: herbáceas perennes, arbustos bajos ( $>1 \mathrm{~m}$ de altura), arbustos medianos ( $>1 \mathrm{~m}$ de altura) y árboles (un solo fuste), que caracterizan las especies vegetales pioneras, las intermedias tempranas, las intermedias tardías y las de la fase madura respectivamente, siguiendo la maduración del ecosistema y considerando el incremento secuencial en la altura de las plantas dominantes. El ensamblaje de especies, caracterizado por la curva de distribución de abundancias relativas, representa la composición y abundancia específicas de una comunidad y muestra el tipo de dominancia (o su ausencia), en el conjunto de especies agrupadas en el mismo hábitat. Por ello, el que exista un ajuste estadísticamente significativo entre fitobiontes y micobiontes, en las cuatro etapas serales, refleja un patrón similar en el tipo de dominancia en ambas comunidades. Así, hay tres etapas serales donde hay una dominancia mono-específica tanto de poblaciones vegetales como de morfo-especies de HMA y que corresponden a los mosaicos de Buchloë dactyloides, Mimosa biuncifera y Prosopis laevigata. En el caso del mosaico caracterizado por Mimosa depauperata, donde se asocia este arbusto bajo con Fluorensia resinosa, hay una codominancia de dos especies tanto en la comunidad de plantas como en la de HMA. En el caso del mosaico de $B$. dactyloides, la especie vegetal con mayor índice de Valor de Importancia es Mimosa depauperata. Esto muestra el papel central que tienen las especies leñosas denominadas nodrizas en la dinámica de un matorral xerófilo y la estrecha relación entre las especies dominantes de hongos micorrizógenos arbusculares y de la vegetación de un hábitat semiárido, durante la sucesión vegetal, lo cual es relevante para la planeación de programas de restauración ambiental de ecosistemas semidesérticos deteriorados.

No se encontró una variación significativa en el porcentaje de colonización micorrízica de gramíneas asociadas a las plantas dominantes, de cada una de cuatro fases sucesionales del matorral xerófilo analizado. Se consideró la maduración del ecosistema siguiendo una secuencia de desarrollo en altura de las poblaciones de plantas, como indicador de la formación de nuevos estratos vegetales. Esto indica que hay una comunidad de HMA desde la fase sucesional temprana (67\% de colonización micorrízica en la fase de plantas herbáceas colonizadoras caracterizada por Buchloë dactyloides), la cual es un factor determinante en el proceso de maduración del ecosistema, al favorecer el establecimiento de plantas leñosas cada vez con mayor biomasa y estructura, tanto aérea como subterránea, mediante la red hifal.

\section{Agradecimientos}

Los autores agradecen las observaciones y aportaciones hechas a este estudio por tres revisores anónimos, así como al Biól. Mariano García Díaz, al M. en C. Eduardo Chimal Sánchez y a la Dra. Rosalva García Sánchez. También, se agradece el financiamiento de este trabajo otorgado por la Dirección General de Asuntos del Personal Académico (DGAPA), de la Universidad Nacional Autónoma de México(UNAM), mediante el proyecto PAPIIT con clave IN218317.

\section{Referencias}

Aguilera Gómez, L.I., Olalde Portugal, V., RubíArriaga, M., \& Contreras Alonso, R. (2007). Micorrizas arbusculares. Ciencia Ergo Sum, 14,300-306. http://www.redalyc.org/pdf/104/10414307.pdf,DOI: 104/10414307.pdf

Alanís-Rodríguez, E., Mora-Olivo, A., Jiménez-Pérez, J., GonzálezTagle, M.A., Yerena Yamallel, J.G., Martínez-Ávalos, J.G. \& González-Rodríguez, L.E. (2015). Composición y diversidad del matorral desértico rosetófilo en dos tipos de suelo en el noreste de México. Acta Botánica Mexicana, 110, 105-117.

Allen, E.B.,Allen, M.F., Egerton-Warburton, L., Corkidi,L., \& GómezPompa, A. (2003). Impacts of early- and late-seral mycorrhizae during restoration in seasonal tropical forest, Mexico. Ecological Applications, 13, 1701-1717. DOI: 10.1890/02-5309 
Allen, E.B. \& Allen, M.F. (1984). Competition between plants of different successional stages: Mycorrhizae as regulators. Canadian Journal of Botany, 62, 2625-2629. DOI:10.1139/b84-356

Allen, M.F.(1991). The ecology of mycorrhizae. (Cambridge University Press, Cambridge). DOI:10.1017/S0266467400006337

Aziz, T., Sylvia, D.M. \& Doren, R.F. (1995). Activity and Species Composition of Arbuscular Mycorrhizal Fungi Following Soil Removal. Ecological Applications, 5, 776-784. DOI: $10.2307 / 1941985$

Barragán Valdez, E.A. (2003). Inoculación micorrízica de Prosopis laevigata (mezquite) en condiciones de invernadero y su efecto al trasplante a condiciones de campo, 67 págs. (Tesis de licenciatura, FES Zaragoza, UNAM, México).

Bethlenfalvay, G.J., Brown, M.S., Ames, R.N. \& Thomas, R.S. (1998). Effects of drought on host and endophyte development in mycorrhizal soybeans in relation to water use and phosphate uptake. Physiologia Plantarum, 72, 565-571. DOI: 10.1111/j.13993054.1988.tb09166.x

Bever, J.D., Morton, J.B., Antonovics, J. \& Schultz, P.A. (1996). Host-Dependent Sporulation and Species Diversity of Arbuscular Mycorrhizal Fungi in a Mown Grassland. Journal of Ecology, 84, 71-82. DOI: $10.2307 / 2261701$

Boddington, C.L. \& Dodd, J.C. (2000). The effect of agricultural practices on the development of indigenous arbuscular mycorrhizal fungi. II. Studies in experimental microcosms. Plant and Soil, 218, 145-157. DOI: $10.1023 / \mathrm{A}: 1014911318284$

Camargo-Ricalde, S.L. \& Esperón-Rodríguez, M. (2005). Efecto de la heterogeneidad espacial y estacional del suelo sobre la abundancia de esporas de hongos micorrizógenos arbusculares en el valle semiárido de Tehuacán-Cuicatlán, México. Revista de Biología Tropical, 53: 339-352.

Campo, A.M. \& Duval, V.S. (2014). Diversidad y valor de importancia para la conservación de la vegetación natural. Parque Nacional Lihué Calel (Argentina). Anales de Geografia, 34, 25-42. DOI: 10.5209/revAGUC.2014.v34.n2.47071

Cervantes, R.M.C. (2002). Plantas de importancia económica en las zonas áridas y semiáridas de México. (Instituto de Geografía, UNAM, México).

Chimal-Sánchez, E., Araiza-Jacinto, M.L. \& Román-Cárdenas, V.J. (2015). El efecto del fuego en la riqueza de especies de hongos micorrizógenos arbusculares asociados a plantas de matorral xerófilo en el Parque Ecológico "Cubitos". TIP Revista Especializada en Ciencias Químico-Biológicas, 18(2), 107-115. DOI: 10.1016/j.recqb.2015.09.002

Chimal-Sánchez, E., García-Sánchez, R. \& Hernández-Cuevas, L.V. (2015). Gran riqueza de hongos micorrizógenos arbusculares en el Valle del Mezquital, Hidalgo, México. Revista Mexicana de Micología, 41: 15-26.

Cottam, G. \& Curtis, J.T. (1956). The Use of Distance Measures in Phytosociological Sampling. Ecology, 37, 451- 460. DOI: $10.2307 / 1930167$

Cruz P., C. (2013). Cuantificación de la colonización intrarradical y extrarradical de hongos micorrizógenos arbusculares en un mosaico heterogéneo en el trópico seco de Veracruz, 95 págs. (Universidad Nacional Autónoma de México, Tesis Maestría, México).

Davies, F.T., Potter, J.R. \& Linderman, R.G. (1992). Mycorrhiza and Repeated Drought Exposure Affect Drought Resistance and Extraradical Hyphae Development of Pepper Plants Independent of Plant Size and Nutrient Content. Journal of Plant Physiologist, 139, 289-294. DOI: 10.1016/S0176-1617(11)80339-1

Di Rienzo, J.A., Casanoves, F., Balzarii, M.G., González, L., Tablada, M. \& Robledo, C.W. (2016). InfoStat. Grupo InfoStat, FCA (Universidad Nacional de Córdoba, Argentina).

Encina, D.J., Meave, A.J. \& Zárate, L.A. (2013). Structure and woody species diversity of the Dasylirion cedrosanum (Nolinaceae) rosette scrub of central and southern Coahuila State, Mexico. Botanical Sciences, 91, 335-347. DOI:10.17129/botsci.12

Escalona, M., Trejo, D., Rivera, J., Lara, L. \& Rivera, A. (1998). Efecto de la endomicorriza arbusculary diferentes fechas de fertilización sobre el crecimiento de papaya en campo. (Memorias de la décima primera reunión científica tecnológica y agropecuaria del estado de Veracruz, México).

Evans, D.G. \& Miller, M.H. (1990). The role of the external mycelial network in the effect of soil disturbance upon vesicular-arbuscular mycorrhizal colonization of maize. New Phytologist, 114, 65-75. DOI: $10.1111 / j .1469-8137.1990 . t b 00374 . x / p d f$

García-Sánchez, R., Camargo-Ricalde, S.L., García-Moya, E., LunaCavazos, M., Romero-Manzanares, A. \& Montaño,A.N.M. (2012). Prosopis laevigata and Mimosa biuncifera (Leguminosae), jointly influence plant diversity and soil fertility of a Mexican semiarid ecosystem. Revista de Biología Tropical, 60, 87-103.

García-Sánchez, R. (2010). Diversidad funcional de los hongos micorrizógenos arbusculares de islas de recurso del Valle del Mezquital, Hidalgo, 112 págs. (Tesis de Doctorado, Colegio de Postgraduados, Campus Montecillo, Postgrado de Botánica, Estado de México).

García-Sánchez, R., Monroy-Ata, A. \& Chimal-Sánchez, E. (2007). en: Micorrizas arbusculares en ecosistemas áridos y semiáridos (Montaño A.N.M., Camargo-Ricalde, S.L., García-Sánchez, R. \& Monroy A., A.) 194-218 (Mundi-Prensa SA de CV, México, Distrito Federal).

Garrido, I., Díaz, R., Escalona, M. \& Trejo, D. (1998). Efecto de la endomicorriza y vermicomposta en plantas de Jamaica en semillero y campo. (Resúmenes del II Simposium Nacional de la Simbiosis Micorrízica, México).

Gerdemann, J.W. \& Nicolson T.H. (1963). Spores of mycorrhizal Endogone species extracted from soil by wet sieving and decanting. Transactions of the British Mycological Society, 46, 235-244. DOI: 10.1016/S0007-1536(63)80079-0

González Medrano, F. (2004). Las comunidades vegetales de México: propuesta para la unificación de la clasificación y nomenclatura de la vegetación de México, 80 págs. (Secretaría de MedioAmbientey Recursos Naturales: Instituto Nacional de Ecología. México, D.F.).

González Monterrubio, C.F., Monroy A., A., García A., E.M., \& Orozco A., M.S. (2005). Influencia de hongos micorrizógenos arbusculares (HMA) en el desarrollo de plántulas de Opuntia streptacantha Lem. sometidas a sequía, en condiciones de invernadero. Tip Revista Especializada en Ciencias QuímicoBiológicas, 8(1), 5-10.

González-Rodríguez, H., Ramírez-Lozano, R., Cantú-Silva, I., Gómez-Meza, M.V. \& Uvalle-Sauceda, J.I. (2010). Composición y estructura de la vegetación en tres sitios del estado de Nuevo León, México. Polibotánica, 29, 91-106. DOI: 62112471004

Grubb, P.J. (1985). Plant Population and Vegetation in Relation to Habitat, Disturbance and Competition: Problems of Generalization. The Population Structure of Vegetation. 3, 595621. DOI: 10.1007/978-94-009-5500-4_25 
Gupta, R. \& Mukerji, K.G. (2000). en: Mycorrhizal Biology (Mukerji K.G., Chamola B.P., Singh J.) 57-65 (Springer Science, New York).

Harley, J. L. \& Smith, S. E. (1983). Mycorrhizal Symbiosis. (Academic Press Inc., New York 1983).

Hernández Ladrón de Guevara, M. (2011). Crecimiento en invernadero de Mimosa biuncifera Benth., inoculada con hongos micorrizógenos arbusculares (HMA) provenientes de matorrales xerófitos del Valle del Mezquital, Hgo., 57 págs. (Tesis de Licenciatura. Universidad Autónoma de México, Facultad de Estudios Superiores Zaragoza, México).

Huerta-Martínez, F.M. \& García-Moya, E. (2004). Diversidad de especies perennes y su relación con el ambiente en un área semiárida del centro de México: implicaciones para la conservación. InterCiencia, 29, 435-444.

Husband, R., Herre, E.A., Turner, S.L., Gallery, R. \& Young, J.P.W. (2002). Molecular diversity of arbuscular mycorrhizal fungi and patterns of host association over time and space in a tropical forest. Molecular Ecology, 11, 2669-2678. DOI: 10.1046/j.1365294X.2002.01647.x

Instituto Nacional de Estadística, Geografía e Informática. (2000). Cuaderno estadístico municipal (Gobierno del Estado de Hidalgo, INEGI, Ayuntamiento constitucional de Santiago de Anaya, Aguascalientes México).

Johnson, N.C. (2010). Resource stoichiometry elucidates the structure and function of arbuscular mycorrhizas across scales. New Phytologist, 185, 631-647. DOI: https://doi.org/10.1111/j.14698137.2009.03110.x

Khalvati, M.A., Hu, Y., Mozafar, A. \& Schmidhalter, U. (2005). Quantification of water uptake by arbuscular mycorrhizal hyphae and its significance for leaf growth, water relations, and gas exchange of barley subjected to drought stress. Plant Biology, 7, 706-712. DOI: $10.1055 / \mathrm{s}-2005-872893$

Laska, G. (2001). The disturbance and vegetation dynamics: a review and an alternative framework. Plant Ecology, 157, 77-99. DOI: 10.1023/A:1013760320805

Luna Camacho, L.A. (2005). Influencia de hongos micorrizógenos arbusculares (HMA) sobre el desarrollo de Agave salmiana (Agavaceae) y Opuntia streptacantha (Cactaceae) en condiciones de invernadero, 92 págs. (Tesis de Licenciatura. FES Zaragoza. UNAM).

Margalef, R. (1972). Homage to Evelyn Hutchinson, or why is there an upper limit to diversity. Transactions of the Connecticut Academy of Arts and Sciences, 44, 211-235.

Mata, M., Treviño, E., Valdecantos, A., Jiménez, J., Aguirre, O., Alanís, E. \& Foroughbackhch, R. (2014). Diversidad y composición vegetal de matorrales en el Valle de Santa Catarina, en el noreste de México. Revista Iberoamericana de Ciencias, 1, 3-15.

Medrano Castañeda, H.I. (2002). Obtención de un inóculo endomicorrícico nativo para un agostadero semiárido degradado de Santiago de Anaya, Hidalgo, 98 págs. (Tesis de licenciatura, FES Zaragoza, UNAM, México).

Miller, R.M. \& Jastrow, J.G. (1992). The role of Mycorrhizal fungi in soil conservation. En: Bethlenfalvay, J.G. \& Linderman, R.G. Mycorrhizae in sustainable agriculture. America Society of Agronomy, 54, 29-44.

Miller, R.M., Reinhardt, D.R \& Jastrow, J.D. (1995). External hyphal production of vesicular-arbuscular mycorrhizal fungi in pasture and tallgrass prairie communities. Oecologia, 103, 17-23. DOI: 10.1007/BF00328420
Miranda, F. (1955). "Formas de vida vegetales y el problema de la delimitación de las zonas áridas de México”. Instituto Mexicano de Recursos Naturales Renovables, 85-119.

Monroy-Ata, A., Peña-Becerril, J.C. \& García-Díaz, M. (2016). Mycorrhizal symbiosis organization of dominant tree Prosopis laevigata (mesquite) in a xeric shrub of central México. en: Recent Advances on Mycorrhizal Fungi (ed. Pagano, M.C.) 35-45. DOI: 10.1007/978-3-319-24355-9 4

Monroy-Ata, A., Estevez-Torres, J., García-Sánchez, R. \& Ríos G., R. (2007). Establecimiento de plantas mediante el uso de micorrizas y de islas de recursos en un matorral xerófilo deteriorado. Boletín de la Sociedad Botánica de México, 80, 49-57. DOI: 57708006

Montaño-Arias, N.M., García-Sánchez R., Ochoa-de la Rosa, G. \& Monroy-Ata,A. (2006). Relación entre la vegetación arbustiva, el mezquite y el suelo de un ecosistema semiárido en México. Terra Latinoamericana, 24, 193-205. DOI: 57311108006

Montaño-Arias, N.M. (2000). Potencialidad de los hongos micorrizógenos arbusculares de las islas de fertilidad de mezquite (Prosopis laevigata) de dos agostaderos semiáridos del Valle de Actopan, México central, un enfoque ecológico para recuperar la vegetación, 150 págs. (Tesis Licenciatura, FES Zaragoza, UNAM, México)

Mora D., C.A., Alanís R., J., Jiménez P., J., González T., M.A., Yerena Y., J.I \& Cuéllar R., L.G. (2013). Estructura, composición florística y diversidad del matorral espinoso tamaulipeco, México. Ecología Aplicada, 12, 29-34. DOI: articulo.oa? id=34129467004

Morrin, P.J.(1999). Community Ecology. (Blackwell Science, Oxford).

Mostacedo, B. \& Fredericksen, T.S. (2000). Manual de métodos básicos de muestreo y análisis en ecología vegetal. (El País, Bolivia).

Mueller-Dombois, D. \& Ellenberg, H. (1974). Aims and methods of vegetation ecology. (John C. Wiley \& Sons, New York).

Muñoz C., A.A. (2013). Consorcios de hongos micorrizógenos arbusculares asociados a seis especies vegetales provenientes de matorral xerófilo. 60 págs. (Tesis Licenciatura Universidad Nacional Autónoma de México, Facultad de Estudios Superiores Zaragoza).

Peña B., J.C. (2002). Influencia de los hongos micorrizógenos arbusculares (HMA) en el establecimiento de Mimosa biuncifera Benth. bajo condiciones de sequía en un invernadero, 64 págs. (Tesis de Licenciatura. FES Zaragoza. UNAM).

Phillips, J.M. \& Hayman, D.S. (1970). Improved procedures for cleaning roots and staining parasitic and vesicular-arbuscular mycorrhizal fungi for rapid assessment of infection. Transactions of the British Mycological Society, 55, 158-161. DOI: 10.1016/ S0007-1536(70)80110-3

Pickett, S.T.A., Collins, S.L. \& Armesto, J.J. (1987). A hierarchical consideration of causes and mechanisms of succession. Vegetatio, 69, 109-114. DOI: 10.1007/BF00038691

Pickett, S.T.A. \& White, P.S. (1985). The ecology of natural disturbance and patch dynamics (eds. Pickett, S.T.A. \& White, P.S.). 371-384 (Academic Press, Florida). DOI: 10.1016/B978-0-12-5545204.50026-5

Ramírez-Lozano, R.G., Domínguez-Gómez, T.G., GonzálezRodríguez, H., Cantú-Silva, I., Gómez-Meza, M.V., SarquísRamírez, J.I. \& Jurado, E. (2013). Composición y diversidad de la vegetación en cuatro sitios del noreste de México. Madera Bosques, 19, 59-72. DOI: 61728317004

Rubio R., H., Cepeda M., P., Borie F., B. \& Contreras A., N. (1997). Efecto de hongos micorrizógenos arbusculares sobre el crecimiento 
de algunas hortalizas en almácigo y posterior trasplante. Agricultura Técnica, 57, 161-168.

Siqueira, J.O., Carbone, C.M.A., Curia, N., Rosadob, S.C. \& Davideb, A.C. (1998). Mycorrhizal colonization and mycotrophic growth of native woody species as related to successional groups in Southeastern Brazil. Forest Ecology and Management, 107, 241-252. DOI: 10.1016/S0378-1127(97)00336-8

Smith, S.E. \& Read, D.J. (1997). Mycorrhizal symbiosis. (Academic Press, Cambridge) DOI: https://doi.org/10.1016/B978-0-12652840-4.X5000-1

Sonco S., R. (2013). Estudio de la diversidad alfa ( $\boldsymbol{\alpha})$ y beta $(\boldsymbol{\beta})$ en tres localidades de un bosque montano en la región de Madidi, La Paz, Bolivia, 154 págs. (Tesis Licenciatura. Universidad Mayor de San Andrés, Bolivia).

Treviño C., J. \& Hernández S., L.G. (2000). Introducción a los matorrales rosetófilos de Querétaro, México. (Memorias de los
Mejores Trabajos del Simposio, México).

Van der Heijden, M.G.A., Boller, T., Wiemken, A. \& Sanders, I.R. (1998). Different arbuscular mycorrhizal fungal species are potential determinants of plant community structure. Ecology, 79, 2082-2091. DOI: 10.1890/0012-9658(1998)079[2082:DA MFSA]2.0.CO;2

Varela,L.\& Trejo, D. (2001). Los hongos micorrizógenos arbusculares como componentes de la biodiversidad del suelo en México. Acta Zoológica Mexicana, 1, 39-51.

Villavicencio N., M.A., Pérez E., B.E. \& Ramírez A., A. (1998). Lista florística del estado de Hidalgo recopilación bibliográfica. (Universidad Autónoma del Estado de Hidalgo, Centro de Investigaciones Biológicas, México).

Wilson, J.B.(1999). Assembly rules in plant communities. in: Ecological assembly rules: perspectives, advances, retreats (eds. Weiher, E. \& Keddy, P). 130-164 (Cambridge University Press, Cambridge). 